Design: This is a retrospective study to summarize the telepathology consultation results from January 2012 to August 2013

Results: The case numbers vary from $1-70$ /month with marked increase in the past 5 months with an average of 63 cases/month. The most cases is hematopathology, accounting for $23 \%$ of total cases, followed by bone/soft tissue pathology and gynecologic/breast pathology. Turnaround time varies depending on subspecialties. Average turnaround time was 6.8 days in 2012 and 4.9 days in $2013(\mathrm{p}<0.05)$. Case distribution and turnaround time is listed in table $1.59 \%$ cases submitted to the consultation were referred by pathologists, $38 \%$ by clinicians and $3 \%$ by patients. Malignant cases account for $67 \%$, benign for $20 \%$, and borderline/neoplasm for $13 \%$. $88 \%$ of cases have a definite diagnosis, $8 \%$ with a favored/suggestive diagnosis, and $4 \%$ with an atypical/uncertain diagnosis. $226(44 \%)$ cases were sent for consultation without primary diagnosis and 294 (56\%) cases with a primary diagnosis or impression. Comparing diagnosis from UPMC with primary diagnosis in the 294 cases, the final diagnoses are the same in $21 \%$, mildly modified in $24 \%$, and significantly modified in $55 \%$, which might affect treatment.

\begin{tabular}{|l||l|l||l||l|}
\hline Categories & 2012 & TAT & 2013 (Jan-Aug) & TAT \\
\hline Bone/soft tissue & 32 & 6.2 & 76 & 4.1 \\
\hline Breast/GYN & 32 & 5.4 & 66 & 4.0 \\
\hline Head/Neck & 17 & 7.7 & 28 & 5.8 \\
\hline Hematopath & 2 & 6.5 & 119 & 5.9 \\
\hline GI & 14 & 7.4 & 29 & 4.2 \\
\hline Liver & 9 & 8.1 & 2 & 6.5 \\
\hline Thoracic & 12 & 5.1 & 15 & 4.2 \\
\hline GU & 11 & 11.4 & 14 & 6.4 \\
\hline Derm/Melanoma & 7 & 3.9 & 18 & 3.2 \\
\hline Neuropath & 5 & 6.0 & 5 & 6.0 \\
\hline Others & 3 & 8.3 & 4 & 4.9 \\
\hline Total & 144 & 6.8 & 376 & \\
\hline
\end{tabular}

Conclusions: Our results indicate that international telepathology consultation can improve patient care and safety through access to pathology expertise. The successful clinical service by international digital pathology depend on many factors, including strong commitment and support from leadership, IT experts, dedicated pathologists who understand language/culture on both sides. Many challenges still remain, such as internet speed, difference in cultures and health care systems, lack of clinical information, difficulties in communication with clinicians. Nevertheless, the internatonal collaboration has had definite positive impact in patient diagnostic care through telepathology.

\section{Kidney/Renal Pathology}

\section{6 \\ qRT-PCR Detection of BK Virus in Renal Biopsy Specimens from Renal Transplant Patients}

RE Alexander, K Post, ZZhao, KMeehan, C Phillips, L Cheng, J Lin. Indiana University School of Medicine, Indianapolis, IN; IU Health, Indianapolis, IN.

Background: BK virus infection in renal transplant patients is a constant concern due to the immunosuppressive therapies used in these patients. As BK-induced nephritis may lead to graft failure, detection of the virus in patients that allows for appropriate alterations in therapy is of significant clinical importance. While the diagnosis of BK virus infection can be made on H\&E stained slides, the use of immunohistochemistry (IHC) has allowed for greater detection with higher specificity. The goal of this study was to compare the diagnostic effectiveness of using qRT-PCR to detect BK virus versus conventional light microscopic and IHC techniques.

Design: 65 renal biopsy specimens from renal transplant patients taken for evaluation of the presence for BK virus were selected. 26 of these cases had definitive histologic and/or IHC evidence of BK virus; the remaining 39 cases lacked sufficient evidence on either H\&E or IHC. DNA extraction was performed on all cases and qRT-PCR was conducted. In addition, this process was also run on a "control group" of 49 renal biopsies taken from non-transplanted and non-immunosuppressed patients to establish specificity. Results: In the 26 cases with definitive BK virus infection by H\&E and/or IHC, qRTPCR detected BK virus in 10 cases ( $62 \%$ sensitivity). In the control group of cases, no evidence of viral infection was shown by qRT-PCR with a specificity of $100 \%$. Of the 39 cases with clinical suspicion of BK virus, but diagnosed as negative by H\&E and/ or IHC, qRT-PCR found 2 cases with BK virus. Analysis of these cases revealed that there was no viral inclusion on H\&E, but no IHC had been performed. Subsequently, IHC was performed and showed focal BK positivity and confirmed the PCR detection. Conclusions: These findings indicate that qRT-PCR is a highly specific detection method of BK virus, although the sensitivity is low when compared to traditional H\&E and IHC methods. In light of these findings, the use of PCR may be particularly useful in cases with equivocal findings on $\mathrm{H} \& \mathrm{E}$ or $\mathrm{IHC}$ that can neither produce a definitive diagnosis or exclusion of virus. Despite its apparent lack of sensitivity, the high specificity of this modality infers its clinical utility in the proper context.

1657 Pulmonary Fibrosis-Renal Lipofuscinosis (“Black Kidney”), a New Disorder with Histologic Features of Hermansky-Pudlak Syndrome N Aljinovic, AR Cullinane, BR Gochuico, WA Gahl, S Rosen. Beth Israel Deaconess Medical Center, Boston, MA; National Institutes of Health, Bethesda, MD; Harvard Medical School, Boston, MA.

Background: Membrane trafficking is an important cellular function and its failure is highlighted in Hermansky-Pudlak Syndrome (HPS), in which the functions of lysosomelike organelles are impaired by biallelic mutations in one of 9 different genes. As a consequence, hypopigmentation is present in the skin, hair, and eyes due to defective maturation of melanosomes, and bleeding occurs due to the absence of platelet dense core granules. In addition, lipofuscin appears especially in the kidney, probably because of defective lysosomal membrane turnover. HPS subtypes 1,2 , and 4 also manifest pulmonary fibrosis. The cause of this is unknown, but the type II pneumocytes have abnormally enlarged and abundant lamellar bodies. The characterization of 15 true mouse models of HPS helped identify murine and human HPS genes, largely through positional cloning. Kidney involvement is not overt in these mouse models, but renal lysosomal abnormalities have been documented both biochemically and by electron microscopy. Renal lipofuscinosis can be seen rarely in humans as an apparently solitary finding, but is common is some animal species, unassociated with kidney dysfunction. Design: We present one family with pulmonary fibrosis and typical pneumocyte changes of HPS, renal lipofuscinosis and no platelet or oculocutaneous pigmentary abnormalities. The family had three brothers. Probands 1 and 2 died of pulmonary disease, and proband 3 has biopsy-confirmed pulmonary fibrosis. Proband 1 donated his kidney for transplantation; the kidney was refused because of its characteristic gross appearance. Results: The lung biopsy in proband 3 showed peribronchial and septal fibrosis, hyperplasia and vacuolization of type II pneumocytes with extensive and giant lamellar body formation (electron microscopy). The explanted kidney of proband 1 showed abundant renal deposition of lipofuscin pigment and whole mount transmission electron microscopy revealed normal platelet dense granules. Genetic studies of proband 3 and two unaffected siblings showed that HPS related genes are not involved, but there is a unique mutation in a distinct gene containing a BEACH domain. Functional studies are underway to assess causality.

Conclusions: We present a new pulmonary-renal entity characterized by a fatal lung disease and marked renal lipofuscinosis, similar to HPS but with a distinct genetic mutation.

\section{Overload of Polyclonal Light Chains in Proximal Tubules Can} Be Associated with Acute Tubular Injury and Interstitial Fibrosis

A Almradi, SC Wolforth, $R$ Parasuraman, $W$ Li, MT Rooney, PL Zhang. William Beaumont Hospital, Royal Oak, MI.

Background: Monoclonal light chains have been known to be nephrotoxic. Although serum levels of free polyclonal light chains (PLC) are found elevated in chronic kidney diseases by several groups, potential damage of PLC in the kidney has not been studied before. Our preliminary study showed that light chain staining by immunohistochemical method (IHC) was similar to that by immunofluorescent method in renal biopsies. This study was to determine whether overexpression of PLC in proximal tubules was associated with both acute renal injury and subsequent chronicity.

Design: Three groups of polyclonal cases included normal renal parenchyma from nephrectomy specimens $(n=39$, normal controls), minimal change disease $(n=13$, MCD controls) and PLC study group composed of random native biopsies with various chronic kidney diseases $(n=33)$. They were all stained for kappa and lambda by IHC and their kappa staining scores ( 0 to $3+$ ) were correlated with serum creatinine levels $(\mathrm{sCr})$, periodic acid-schiff stain (PAS) scores for diminished brush borders ( 0 to $3+$ ), and fibrosis scores on Trichrome stained sections $(0$ to $3+)$ using linear regression analysis. Results: The two control groups had significant lower PAS and fibrosis scores when compared to PLC study group (by ANOVA). Taken 3 groups together, there were significant correlations between either sCr, PAS scores or fibrosis scores (on Y axis), and kappa scores (on X axis) as shown in the following table $\left({ }^{*} \mathrm{p}<0.05=\right.$ significance).

\begin{tabular}{|l|l|l|l||}
\hline Total $\mathbf{n}=\mathbf{8 5}$ & $\begin{array}{l}\text { Kappa scores } \\
\text { vs. } \mathbf{~} \mathbf{C r}\end{array}$ & $\begin{array}{l}\text { Kappa scores vs. PAS } \\
\text { scores }\end{array}$ & $\begin{array}{l}\text { Kappa scores vs. Fibrosis } \\
\text { scores }\end{array}$ \\
\hline R value & 0.42 & 0.54 & 0.66 \\
\hline Odds ratio & 1.75 & 0.76 & 0.66 \\
\hline $\begin{array}{l}\text { 95\% confidence } \\
\text { interval }\end{array}$ & 1.52 to 2.02 & 0.62 to 0.81 & 0.51 to 0.81 \\
\hline P value & $0.0001^{*}$ & $0.0001^{*}$ & $0.0001^{*}$ \\
\hline
\end{tabular}

Conclusions: Our data, showing significant association of PLC staining in the proximal tubules with acute and chronic renal injury, suggest that chronic overload of freely filtered PLC (due to their low molecular weights via glomerular filter barrier) in the proximal tubules can be nephrotoxic.

1659 Kikuchi Disease-Like Interstitial Necroinflammation in Renal Biopsies Associated with Autoimmune Disease: A Report of Three Cases $J$ Ambruzs, LN Cossey, R Lorsbach, CP Larsen. Nephropath, Little Rock, AR; University of Arkansas for Medical Sciences, Little Rock, AR.

Background: Kikuchi Disease (KD) is a rare, self-limiting disorder primarily involving lymph nodes and characterized by necrotizing histiocytic inflammation with prominent apoptotic debris. The etiology is unknown and few reports have shown an association with autoimmune disease but extranodal involvement has been described. We report the first cases of a KD-like necroinflammatory process occurring in the kidney in patients with autoimmune disease.

Design: We identified 3 native renal biopsies from our database with a primary finding of necrotizing lymphohistiocytic interstitial inflammation without evidence of infection or lymphoproliferative disorder.

Results: The cases involved two females and one male (14-40 years). Ethnicities included Native American, African American and Caucasian. All patients presented with fever, neutropenia, anemia, and elevated liver enzymes. One had documented lymphadenopathy. One patient had a history of mixed connective tissue disease while two had systemic lupus erythematosus. All cases had proteinuria, two had concurrent hematuria, and one had acute kidney injury. The most striking finding on renal biopsy was focal interstitial inflammation and necrosis characterized by amorphous eosinophilic debris and prominent apoptotic debris. All cases showed a predominantly T-cell lymphocytic and histiocytic infiltrate. A majority of T-cells were of the CD8+ cytotoxic type in two cases and scattered histiocytes showed MPO positivity. AFB and GMS stains were negative as was in-situ hybridization for EBV in all cases. There was mild, concurrent glomerular immune-complex deposition in two cases with no 
significant glomerular findings in the third. In follow-up patient 1 died secondary to respiratory distress 2 years after diagnosis but had no renal progression. Patient 2 developed nephrotic range proteinuria 5 years after biopsy, but renal function remained stable. Patient 3 is a recent diagnosis and is undergoing treatment.

Conclusions: Herein we report the first cases of a KD-like necroinflammatory process occurring in the kidney. All cases involved patients with autoimmune disease receiving immunosuppressive therapy while none showed renal progression. These findings suggest that extranodal KD (or a morphologically similar process) can occur in the kidneys of patients with autoimmune disease undergoing treatment. Although rare, this entity may be under recognized and further studies better characterizing etiology, risk factors and frequency of renal involvement are needed.

1660 Phenotypic Plasticity of Podocytes and Parietal Epithelial Cells in Diabetic Nephropathy

NK Andeen, TQ Nguyen, F Steegh, KL Hudkins, B Najafian, CE Alpers. University of Washington, Seattle, WA.

Background: Reversal of diabetic nephropathy (DN) has been achieved in humans (Fioretto et al. NEJM 1998) and mice (Pichaiwong et al. JASN 2013). Progression of DN is associated with podocyte (podo) loss; reversal of DN requires restoration of podos. We sought to identify and quantify potential glomerular progenitor cells that could be a source for restored podos.

Design: We selected 31 cases of DN identified in renal biopsies that could be separated into morphologically early (class I, II) or advanced (class III, IV) lesions, corresponding to recent classification of DN (Tervaert et al JASN 2010). Immunohistochemistry was performed for markers of podos (WT-1, p57), parietal epithelial cells (PECs: claudin1) and proliferation (Ki-67) to test the hypothesis that some cells classically considered PECs by location could serve as progenitor cells for restoration of lost podos. Cells located on Bowman's capsule were considered PECs; cells located on the glomerular tuft were considered podos.

Results: WT-1-positive podos are reduced in DN. p57 confirmed reduction of podos, and demonstrated increased p57-positive cells in PEC locations in DN compared to controls. Claudin1-positive cells in podo locations were significantly increased in advanced DN vs controls. Proliferating cells in both podo and PEC locations were identified in $\mathrm{DN}$, but not in controls $(\mathrm{p}<0.05)$.
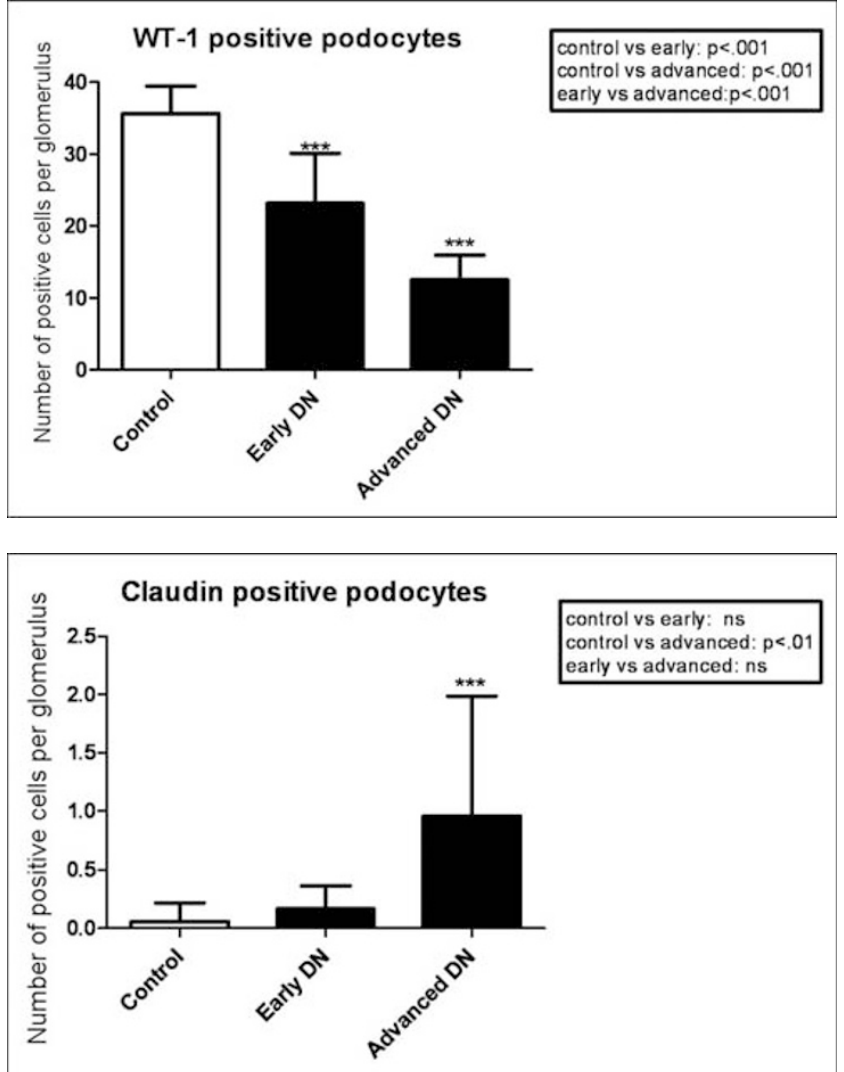

Conclusions: These findings suggest activity and plasticity in podocyte and parietal epithelial cell populations that potentially enable repair in diabetic nephropathy. Reduction in podo number by WT- 1 and p57 immunolabeling confirms previous studies in type I and type II associated DN. Increased proliferation and expression of podocyte marker p57 in PECs in DN are consistent with studies in the BTBR ob/ob murine model of reversible DN, and support a hypothesis that some low level of regeneration from a PEC niche may be ongoing in DN which could be increased by new therapeutics for DN.
1661 Expression of the Multi-PDZ Domain Scaffold Protein MAGI-2 Is Necessary for Maintenance of Glomerular Slit Diaphragm Integrity

MD Balbas, MR Burgess, R Murali, J Wongvipat, BJ Skaggs, P Mundel, CL Sawyers. Memorial Sloan-Kettering Cancer Center, New York, NY; University of California, San Francisco, San Francisco, CA; University of California, Los Angeles, Los Angeles, CA; Harvard Medical School and Massachusetts General Hospital, Boston, MA.

Background: The podocyte slit diaphragm is a cell-cell junction that controls urinary filtration in the glomeruli. Nephrin is a transmembrane signaling receptor that is expressed at cell junctions between interdigitating podocyte foot processes. With other slit diaphragm proteins (NEPH1, NEPH2), nephrin forms a zipper-like structure that prevents proteins from entering the glomerular urinary space. Mutations in the gene that encodes nephrin (NPHSI) are seen in severe congenital nephrotic syndrome and in patients with focal and segmental glomerulosclerosis (FSGS). Many interacting partners of nephrin (e.g. podocin, $\alpha$-actinin-4, CD2AP, NEPH1) are also essential for proper glomerular function. Recent work identified the scaffolding protein MAGI-2 as a nephrin interacting partner and member of the glomerular slit diaphragm protein complex. The aim of this study was to characterize the biological function of MAGI-2 by generating Magi-2 knockout mice.

Design: Briefly, we disrupted the Magi-2 gene by homologous recombination in 129 LW1 embryonic stem (ES) cells by replacing a genomic fragment of exon 4 (Magi-2 transcript variant 1) with a neomycin resistance cassette. ES cells were injected into C57Bl/6 (B6) blastocysts and resulting male chimeras were crossed with B6 or 129/ SvJ females. Offspring were genotyped initially by Southern blot and subsequently by PCR. Heterozygous Magi-2 $2^{t m I M b+}$ mice were intercrossed to generate Magi-2 $2^{2 \mathrm{~m} \mid \mathrm{Mb} / \mathrm{m} I \mathrm{Mb}}$ (MAGI-2 knockout) mice.

Results: Loss of MAGI-2 expression resulted in proteinuria and FSGS. The onset of glomerular dysfunction and proteinuria began within 2 weeks of birth. Glomerulosclerosis was histologically evident by age 5 weeks, and increased in extent and severity thereafter. In Magi-2-null mice, the onset of proteinuria coincided with loss of nephrin protein expression. Transmission electron microscopy of kidneys of Magi-2-null mice aged 4 weeks revealed almost complete effacement of glomerular podocyte foot processes.

Conclusions: MAGI-2 expression is essential to maintain proper function of the slit diaphragm. The MAGI-2 knockout mouse is a new model for renal diseases characterized by podocyte effacement (such as FSGS), and may help further our understanding of nephrotic syndromes of unknown molecular etiology.

1662 Molecular Profiling of Glomeruli in a Glomerular Hypertension Mouse Model

M Belghasem, P Bondzie, M Cao, T Norman, J Henderson. Boston University School of Medicine, Boston, MA.

Background: Podocytes contribute to the intricate structure of the glomerular filtration barrier, and likely play a critical role in bolstering the glomerular capillary walls against hemodynamic pressure. Glomerular hypertension has been shown to have a deleterious effect on podocytes and is a major determinant of progression to end-stage renal failure. In vitro studies have shown that podocytes respond to mechanical stress. However, the molecular alterations of podocytes in glomerular hypertension are not well understood. In this study, we characterized changes in gene expression in glomeruli at different time points using a mouse model of glomerular hypertension.

Design: Hypertension was induced in 10 week old $129 \mathrm{~Sv}$ mice by uninephrectomy and subcutaneous implantation of deoxycorticosterone time release tablets. Mice were given $1 \%$ salt solution as drinking water, systolic blood pressure was measured by the tail cuff method, and urine was collected and analyzed for albumin by ELISA (Exocell). At 1 day, 1 week, and 6 weeks post-op, the residual kidney was harvested and high-purity glomerular samples were isolated for RNA extraction. Additional kidney tissue was retained intact for morphologic examination. Microarrays were used to measure global gene expression levels using Illumina MouseWG-6 v2.0 Beadchips. Differentially expressed genes were identified using the lumi and limma packages of $\mathrm{R} /$ Bioconductor. Ingenuity Pathway Analysis was used to identify the most significant biological functions represented among the differentially expressed genes.

Results: Within 1 week post-op, mice developed hypertension and proteinuria. At 6 weeks post-op, multi-focal global and segmental glomerulosclerosis was observed, along with multi-focal tubulointerstitial chronic damage. Gene expression analysis discovered 497 differentially expressed genes with a cutoff of $p<0.05$ and $\log$ fold change $>0.5$. The top canonical pathways associated with this gene set were protein ubiquitination, GADD45 signaling, and VDR/RXR activation pathways. Of note, the transcript for small heat shock proteins was strongly up-regulated at early time points. Conclusions: Glomerular damage observed in the DOCA-salt uninephrectomy model of glomerular hypertension is preceded by changes in glomerulargene expression involving protein ubiquitination, GADD45 signaling, and VDR/RXR activation. In particular, small heat shock proteins are strongly up-regulated early on, consistent with in vitro findings in stretched podocytes, and suggesting alterations of protein synthesis/degradation.

1663 The T-Statistic Is a Robust Statistic for Estimating the Size of Glomeruli in Small Renal Biopsies

$J$ Benhamida, G Szabo, ZG Laszik. UC San Francisco, San Francisco, CA.

Background: Quantitative glomerular parameters, such as size, volume, number, etc. correlate with disease states and physiologic variables with well-established diagnostic and prognostic relevance. However, the accuracy and precision of estimates from biopsies with small numbers of glomeruli is uncertain because the underlying distributions are not known a priori. The Student's t-distribution is robust for large enough sample sizes when the population is not normally distributed. In this study 
we characterize the size distribution of glomeruli and the utility of the Student's t-distribution for estimating the mean glomerular size in 63 nephrectomy specimens using whole slide digital images (WSDI) and computer-assisted quantitative image analysis.

Design: Whole slide digital images were generated from H\&E stained sections from the tumor-free portion of formalin fixed paraffin embedded non-diabetic non-hypertensive nephrectomy specimens ( $\mathrm{n}=63$, median age 53 years) with renal cell carcinoma. No significant histologic abnormalities were present. An Aperio platform was used to measure the size of the glomeruli (in $\mu \mathrm{m}^{2}$, mean $\mathrm{n}=258$ glomeruli/case). For each specimen, a sampling distribution of means was constructed for each sample size between 5 and 50 by generating 1,000 random samples using Monte Carlo sampling. Q-Q plots were constructed to assess the convergence of the t-statistic distribution to the t-distribution.

Results: Across all patients, the mean glomerular size was $6,328 \mu \mathrm{m}^{2}$ (standard deviation $1,222 \mu^{2}$ ) and the mean coefficient of variation was 0.56 (standard deviation 0.14 ). The size distributions of glomeruli vary significantly across patients and are decidedly non-normal. Despite this, Q-Q plots of the t-statistic distribution rapidly converge to the t-distribution with increasing sample size. For moderately skewed distributions, a sample size between 10 - 20 was sufficient to ensure convergence. Highly skewed size distributions, however, required sample sizes between 25-30 for convergence. Conclusions: The distribution of glomerular size varies markedly between individuals Some are moderately symmetric and others are highly skewed. Despite non-normality, the simulated t-statistic distributions approach the t-distribution for sample sizes reasonably obtained in small biopsies. This data supports the robustness of the t-statistic for estimation and statistical testing of glomerular size from biopsies that contain at least 25 glomeruli.

1664 Endothelial Electron Microscopy Scoring and Endothelial Gene Expression Are Biomarkers of Poor Kidney Transplant Survival in Patients with Transplant Glomerulopathy

PL Blanco, S Husain, B Sis. The Ottawa Hospital and University of Ottawa, Ottawa, ON, Canada; University of Alberta Hospital, Edmonton, AB, Canada.

Background: Transplant glomerulopathy (TG) is a sign of chronic kidney transplant damage with poor survival and no effective therapies. However, not all patients with TG progress to graft failure and some patients stay free of dialysis for years. There are significant limitations in the understanding of disease progression in TG. We aimed to explore genome-wide microarray gene transcription profiles of TG and their relationship with ultrastructural changes in kidney biopsies and graft survival.

Design: The study population included kidney allograft biopsies for cause from 40 patients with TG and post-transplant time matched 54 control patients without TG. We developed an electron microscopical (EM) scoring system to semi-quantify individual endothelial changes in glomeruli and peritubular capillaries: cytoplasmic swelling, loss of fenestrations, subendothelial widening, microvilli, and podocyte changes: cytoplasmic swelling and foot process effacement, and recorded a sum-score for endothelial cells and podocytes.

Results: A class comparison showed 34 probe sets as higher in TG vs. non-TG kidneys, including 7 endothelial transcripts (i.e. DARC, VWF, MCAM, ICAM1) and 6 Fc receptor transcripts (i.e. FCGR3A,B, FCGR2A, FCGR1B, FCER1G), suggesting that endothelial changes and NK cells/macrophages are involved in pathogenesis of TG. Microvascular endothelial EM sum-scores were higher in TG versus non-TG biopsies and positively correlated with molecular disturbances, particularly transcripts reflecting endothelial activation, NK cell and interferon-gamma effects $(\mathrm{p}<0.001)$. Podocyte EM sum-scores were not different between TG and non-TG. We stratified TG into 2 prognostic groups using either molecularly or electron microscopically defined endothelial activation: progressive TG kidneys with accelerated graft loss and nonprogressive TG kidneys with no significant graft failure. Of progressive TG cases, only $37 \%$ were $\mathrm{C} 4 \mathrm{~d}$ positive. In multivariate prediction models, endothelial EM sum-score and endothelial gene expression were independent prognostic factors after adjusting for clinical and pathological confounders.

Conclusions: Increased renal endothelial stress is related to progression of TG and endothelial EM scoring or endothelial gene expression can serve as predictive biomarkers for poor kidney transplant survival in patients with TG.

1665 Renal Biopsy in Patients $\mathbf{8 0}$ and Older: Fruitful or Futile?

CL Boils, PD Walker. Nephropath, Little Rock, AR.

Background: We have noted a marked increase in renal biopsies from the very elderly, likely due to a healthier aging population, and that renal biopsies have become safer, with higher yield, along with better treatments for various renal diseases. The aim of this study was to examine the diagnostic utility of the renal biopsy in the very elderly based on the finding of important therapeutic information.

Design: All native renal biopsies received from March 2001 through February 2013 were reviewed and biopsy findings in the very elderly (VE, aged 80 years and older) and the elderly (E, patients aged 60 to 79 years) were compared.

Results: During this twelve-year period, 37924 native renal biopsies were identified with $1549(4.1 \%)$ from the very elderly and $11474(30 \%)$ from the elderly. $<2 \%$ had insufficient material for diagnosis. Crescentic glomerulonephritis was the most common glomerular disease in the very elderly (VE:E, 12\%:8\%) and diabetic nephropathy the most common in the elderly (VE:E, 10\%:17\%). Pauci-immune type (VE:E, 11\%:7.3\%) and anti-glomerular basement membrane antibody type (VE:E, 0.9\%:0.28\%) were significantly more common in the very elderly $(\mathrm{p}=<0.0002)$; immune-complex type (VE:E, $0.45 \%: 0.62 \%$ ) was more common in the elderly ( $<<0.5$ ). Diagnoses statistically more common in the very elderly included acute tubular injury (VE:E, 22\%:19\%, $\mathrm{p}<0.01$ ), light chain cast nephropathy (VE:E, $3.2 \%: 2.4 \%$, p $<0.04$ ), and acute interstitial nephritis (VE:E, $5.5 \%: 4.1 \%$, p $<0.02$ ). Of diseases known to cause nephrotic syndrome,
FSGS was the most common diagnosis in both (VE:E, 11\%:12\%), followed by membranous glomerulopathy (MG, VE:E, 4.9\%:7.6\%) and minimal change disease (VE:E, 3.6\%:3.0\%). Interestingly, MG was significantly less common in the very elderly ( $<<0.001$ ) as was diabetic glomerulopathy (VE:E, 10\%:17\%, $<<0.001$ ), fibrillary glomerulopathy (VE:E, 0.52\%:1.3\%, p<0.01) and lupus nephritis (VE:E, 0.1\%: 1.2\%, $\mathrm{p}<0.01$ ). Additional VE renal biopsy findings of importance included IgA nephropathy (4.8\%), amyloidosis (4.7\%), light chain deposition disease (2.7\%), MPGN $(2.3 \%)$, and proliferative and exudative glomerulonephritis $(0.9 \%)$. Perhaps the most important finding was the arterionephrosclerosis only group ( $<20 \%$ in VE and E), useful more for what was not present, since no change in therapy was indicated.

Conclusions: Biopsy findings of critical significance were commonly found in renal biopsies from the VE, similar in importance to those found in the E and thus "fruitful" not "futile".

1666 Tuberous Sclerosis: The Spectrum of Metanephric Defects, Cystic Kidney Diseases and Neoplasms in 26 Pediatric and Adult Cases

$C$ Boils, $P$ Baker, $M$ Edelman, $N$ Gokden, D Grignon, X Gu, D Holanda, A Kats, A Magil, G Martignoni, S Nasr, D Perry, C Phillips, P Zhang, S Bonsib. Nephropath, Little Rock, AR; Nationwide Children's Hospital, Columbus, OH; Long Island Jewish Medical Center, New York, NY; University of Arkansas, Little Rock, AR; Indiana University, Indianapolis, IN; Louisiana State University, Shreveport, LA; University of Iowa, Iowa City, IA; Children's Mercy Hospital, Kansas City, MO; St. Paul's Hospital, Vancouver, BC, Canada; Universita degli Studi di Verona, Verona, Italy; Mayo Clinic, Rochester, MN; Children's Hospital, Omaha, NE; William Beaumont Hospital, Royal Oak, MI. Background: Tuberous sclerosis (TS), an autosomal dominant disease affects multiple organs, brain and kidneys most often. Renal failure is common, attributable to polycystic cystic kidney disease (PKD) and neoplasms, but other lesions occur. The full spectrum of renal disease in children and adults has not been reported.

Design: 26 cases of TS were obtained from the Jay Bernstein Consultative Collection (17) and files of several authors (9). There are 17 pediatric cases (1 wk-14 yrs) and 9 adult cases (21-64 yrs). Demographic and clinical information was available on 20/26 cases. Results: Four types of lesions occured. (1) metanephric defects (MD) (2) ectatic tubules (ET) and PKDs (3) neoplasms (4) myoid and non-tumor proliferations designated angiomyolipomatosis (AMLosis). MD ( 9 cases) consisted of cortical hypoplasia, medullary islands within PKD, glomerular cysts and dimorphism. ET were lined by large eosinophilic cells (EC). The PKDs (17 cases) included proliferative PKD lined by large $\mathrm{EC}$ in neonates and infants, autosomal dominant-like PKD in older children and adults, and acquired cystic kidney disease in a diabetic with TS. Renal neoplasms ( 8 cases) included multifocal/bilateral angiomyolipomas (AML) and renal cell carcinomas. They occured in adolescents and adults ranging in size from $13 \mathrm{~cm}$ to microscopic. Myoid proliferations enveloped tubules and cysts and were in the interstitium. AMLosis consists of myoid and lipoid interstitial proliferations. Most cases contained more than one lesion. Conclusions: 1) TS has diverse renal manifestations: MD, PKDs, neoplasms, and mesenchymal proliferations 2) MD in the young are associated with proliferative PKD 3) Neoplasms occur in adolescents and adults 4) AMLosis, multifocal AMLs, and EC in tubules and cysts appear unique to TS 5) Familiarity with these lesions is important because $60 \%$ of cases are new mutations and renal disease may be the initial or only manifestation of TS.

\section{Glomerular Gene Expression Profiling and Kidney Morphology in} Carriers of APOL1 Risk Alleles

P Bondzie, T Norman, $S$ Yang, JM Henderson. Boston University School of Medicine, Boston, MA

Background: Mutations in apolipoprotein L1 (APOL1) have been linked to the increased incidence of chronic kidney disease in people of African ancestry. However, little is known about how APOL1 disease risk alleles G1 and G2 contribute to renal dysfunction. Here we hypothesize that carriers of APOL1 risk alleles may exhibit altered kidney morphology and/or glomerular gene expression profiles, which are present before overt kidney injury is apparent. Such changes might favor progression of kidney damage in the face of external stimuli such as hypertension or HIV.

Design: For this IRB approved study, we retrieved formalin-fixed paraffin embedded (FFPE) nephrectomy samples from 51 archived pathology cases available in the BUMC Department of Pathology. DNA extracted from the FFPE samples was used to determine the APOL1 genotype. Samples were stratified into control variants (carriers of at most one APOL1 risk allele) or risk variants (G1G1, G2G2, or G1G2 genotypes). Light microscopic assessment of kidney morphology was performed on $\mathrm{H} \& \mathrm{E}$ and PAS-stained sections of non-lesional kidney. RNA was extracted from laser-captured glomeruli taken from sections of the FFPE samples, and whole genome expression analysis was performed using the Affymetrix Human Gene 1.0 ST array platform. Differentially expressed genes between risk and control variants were analyzed for functional significance using the Ingenuity Pathway Analysis (IPA) software. Results: Samples harboring APOL1 risk variants did not show significant differences in glomerular, tubular or vascular morphology when compared to control samples. However, 558 genes were found to be differentially expressed between risk and control variants using $p$-value $<0.05$ and a minimum fold change of 1.2. Among these genes, strong pathway associations were found relating to kidney failure, organismal injury and disorders, and renal and urologic disease. Strong functional associations relating to cellular movement, cell cycle regulation, cell-cell signaling and interaction, cell death and survival, and cellular assembly and organization were also identified.

Conclusions: Carriers of APOL1 risk variants do not exhibit differences in non-lesional kidney structure at the light microscopic level, compared to control variants. However, glomerular gene expression differences are observed among APOL1 risk variants. The 
strongest pathway associations among the identified differentially expressed genes were seen in pathways associated with kidney injury, potentially explaining why APOL1 risk variant carriers are more susceptible to chronic kidney disease.

1668 Spectrum of Renal Pathology Findings in Hematopoietic Cell Transplant Patients

BT Brinkerhoff, DC Houghton, ML Troxell. OHSU, Portland, OR.

Background: Hematopoietic cell transplant (HCT) patients are exposed to many nephrotoxic insults, including cytotoxic conditioning agents, radiation, infection, immunosuppressive agents, ischemia, and graft versus host disease (GVHD). We reviewed our single center experience.

Design: The pathology database was searched for renal specimens (2007-2013) from patients with history of HCT. Eleven biopsy cases were identified from 10 patients, average 5 years post-HCT (range: 1 month to 19 years). Three additional patients with HCT and subsequent nephrectomy (11-21 years post-HCT) were compared with 11 control nephrectomies. Pathologic findings were correlated with clinical data, when available.

Results: Indications for HCT included hematologic malignancies ( 9 patients: AML, ALL, MDS, myeloma), chronic granulomatous disease (1), and seminoma (1). Seven patients received bone marrow stem cells, 4 received peripheral stem cells, and 1 received cord blood; 3 were autologous, and 9 allogeneic, all 9 with history of GVHD. Clinical indications for biopsy/nephrectomy included nephrotic range proteinuria (7), acute kidney injury (4), renal cell carcinoma (RCC, 2), and chronic ureteral obstruction (1). A diversity of pathologies was found on biopsy: FSGS or podocyte injury (5) including collapsing glomerulopathy and minimal change, acute or chronic thrombotic microangiopathy (TMA, 3), anti-GBM disease (1), amyloidosis (1), and acute tubular injury (ATN, 1). One case with podocyte injury had interstitial nephritis (IN), and a case with TMA also had segmental membranous deposits. The patient with ATN at 1 month post-HCT had a second biopsy at 11 months with TMA and ongoing ATN. Both nephrectomies with RCC had up to $10 \%$ glomeruli with unusual segmental hypercellular sclerosing lesions, while the patient with ureteral obstruction, and 11 control nephrectomies, had no such lesions. Post-biopsy/nephrectomy treatment was mostly conservative (10), while some patients received immunosuppression $(2$, for minimal change/IN) or chemotherapy ( 1 , for myeloma). Outcomes included improved (4) or stable serum creatinine (5), worsening serum creatinine (4), or end stage renal disease (1). One patient died shortly after biopsy (acute TMA).

Conclusions: This single center series highlights a broad spectrum of post-HCT renal pathology, illustrating the value of biopsy in this setting. We report novel findings of collapsing glomerulopathy and anti-GBM disease in the setting of previous HCT. In addition, we report distinctive incidental segmental sclerosing glomerular lesions in 2 BMT patients with nephrectomy for RCC.

\section{Podocyte Loss in Diabetic Glomerulosclerosis}

YM Chen, Y Zhou, J Gaut, H Liapis. Washington University School of Medicine in St. Louis, St. Louis, MO.

Background: Diabetic nephropathy is thought to derive from direct exposure of glomeruli to hyperglycemia and hyperlipidemia. In vitro and in vivo studies of experimental diabetes suggest that podocyte injury and loss is a major mechanism leading to progressive disease. For example, high glucose induces apoptotic podocyte death detected by biochemical assays. Additionally, podocytes exposed to free fatty acids (FFA) show altered podocyte gene expression accompanied by endoplasmic reticulum (ER) oxidative stress. This leads to an unfolded protein response detected by the induction of the ER chaperone immunoglobulin binding protein (BiP). There is little known about how podocytes die in human diabetic nephropathy and whether ER stress and apoptosis occur. We use immunohistochemistry to study diabetic podocytes in renal biopsies and correlate the results with electron microscopy.

Design: Thirty consecutive glomeruli from formalin fixed paraffin embedded renal biopsies $(n=4)$ with diabetic glomerulosclerosis (class III) and normal kidney control glomeruli $(\mathrm{n}=30)$ were evaluated. Diabetic patients had low glomerular filtration rate (GFR) $\left(<30 \mathrm{ml} / \mathrm{min} / 1.73 \mathrm{~m}^{2}\right)$ consistent with chronic kidney disease (CKD) stage 4-5. Mean patient age was 49.5 years. We used immunohistochemistry with an anti-WT1 antibody (Santa Cruz) which labels podocyte nuclei to count podocytes, and an anti$\mathrm{BiP}$ antibody (Lifespan Biosciences) to evaluate ER stress. Retrospective review of electron microscopy was performed.

Results: Diabetic glomeruli contained a mean 9.5 WT1 positive podocytes a fourfold decrease compared to normal glomeruli which had a mean of 44.8 WT1 positive podocytes. BiP was diffusely positive in podocytes of diabetic glomeruli affecting approximately $75 \%$ of podocytes per glomerulus and negative in normal control. Ultrastructural evaluation showed decreased podocyte number, podocyte hypertrophy, cytoplasmic lipid accumulation but absence of apoptotic podocytes.

Conclusions: Diabetic glomeruli show marked podocyte loss and podocyte ER stress induction in class III diabetic nephropathy. The results suggest ER stress may play a role in the process of diabetic glomerulosclerosis, but whether it is involved in loss or survival of diabetic podocytes needs further investigation.

\section{Podocyte Death in Glomerulonephritis}

J Chrisinger, J Gaut, S Jain, H Liapis. Washington University School of Medicine in St. Louis, St. Louis, MO.

Background: Podocytes are terminally differentiated cells arrested in a postmitotic state, but do not have an infinite life span. In fact podocyte loss in the urine is documented. Proteinuria and other injuries accelerate podocyte loss and induce death. There are currently over 10 types of cell death described including apoptosis and mitotic catastrophe, the former characterized by condensed and fragmented nuclei, and the latter defined as podocyte death during mitosis. Whether apoptosis and mitotic catastrophe are indeed a frequent form of podocyte death in human glomerular disease is unknown. Design: We retrospectively reviewed the light and electron microscopy of 250 consecutive renal biopsies for features of apoptosis or podocyte mitosis, i.e. anaphase mitotic figures or multinucleated podocytes implying aneuploidy. All cases had glomerular disorders with significant proteinuria. In a subset of cases including FSGS, MCD, Diabetes, post-infectious glomerulonephritis, MPGN, and Collapsing glomerulopathy (CGP) we performed TUNEL staining using a case of acute tubular necrosis (ATN) as a control.

Results: A total of 18 biopsies (7.5\%) showed podocytes with either multinucleated podocytes or mitotic spindles detected on light or electron microscopy; $4 / 18$ cases were CGP secondary to HIV. We identified only one case of congenital nephrotic syndrome with diffuse mesangial sclerosis to have glomeruli with apoptotic podocytes. TUNEL staining was negative in all cases except ATN (+ control).

Conclusions: The findings suggest that nephrotic syndrome is commonly associated with aneuploid podocytes resulting from aberrant nuclear division without cytokinesis, a characteristic of mitotic catastrophe that eventually leads to podocyte death. Podocyte loss through mitotic catastrophe may be a target for specific podocyte therapies aiming to halt podocyte loss in proteinuria.

\section{Vascular Mesangial 'Shunts' in Nodular Diabetic Glomerulosclerosis} LN Cossey, R Hennigar, S Bonsib, AM Gown, FG Silva. Nephropath, Little Rock, AR; PhenoPath Laboratories, Seattle, WA; University of Arkansas for Medical Sciences, Little Rock, AR.

Background: Channels within the mesangial tree were characterized by Latta and colleagues in 1960 and have been reported in various forms of nodular glomerulosclerosis. The aim of this study is to describe the light and electron microscopic features of these channels and delineate the immunohistochemical phenotype of their lining cells.

Design: Thirty-eight cases of nodular diabetic glomerulopathy (DG) (Tervaert class III or greater) with identifiable mesangial channels (MC) were prospectively selected over a 2.5 year period. All cases were studied by light, electron microscopy and CD31/PAS dual stain. Select cases were immunostained for CD34, podoplanin and ERG, using conventional immunoperoxidase techniques.

Results: Light microscopic analysis confirmed the presence of MC that were randomly distributed within the diabetic nodules. Occasional MC contained RBCs within their lumens, suggestive of vascular spaces. The lining cells showed a typical vascular phenotype expressing CD31, CD34 and ERG. Staining for lymphatic channel marker podoplanin was negative. Mesangial nodules frequently contained CD31/CD34/ ERG-positive cells embedded within the matrix but not lining any obvious channels. In areas of severe mesangiolysis diffuse, granular CD31/34 staining was identified in the absence of vascular spaces. Dual staining with CD31/PAS aided in mapping the course of mesangial channels and showed that channels branch to form direct communications with peripheral capillary loops, suggestive of shunting. These smaller shunts occasionally connected with larger mesangial channels that on longitudinal section, appeared to extend from the periphery of the tuft and feed directly into hilar arterioles. Electron microscopy (EM) of cells lining the shunts was non-specific as to cell of origin. No basement membranes were identified surrounding the channels. Contouring of the channels were irregular and often angulated.

Conclusions: MC present within DG are visible by light microscopy and display a typical vascular phenotype. They connect with peripheral capillary loops and extend deep within the mesangium where they appear to form part of an elaborate shunting network within the glomerulus. We postulate that they arise from endothelial cells that have migrated into the mesangium and that altered blood flow through the glomerulus exerts physiologic pressure on the entrapped cells to undergo angiogenesis and create collateral circulation (or shunts) through the obstructive nodules, attempting to redirect blood flow.

1672 The Extra-Tubular Compartment Is the Main Source of Intragraft IL-6 mRNA Expression in Allograft Kidneys with Acute Cellular and Chronic Antibody-Mediated Rejection

JA Cotter, G Szabo, FG Vincenti, ZG Laszik. University of California San Francisco, San Francisco, CA.

Background: IL-6 is a pleiotropic cytokine with immunomodulatory functions promoting inflammation and inhibiting the suppressive function of natural $\mathrm{T}$ regulatory cells. Recent in vivo experimental data in various models of mouse cardiac transplantation indicate an important role for IL-6 in transplant rejection. Although IL-6 transcripts have been shown to correlate with inflammation and fibrosis in human allograft kidney biopsies, the precise cellular source of IL-6 in transplant kidneys remains to be determined.

Design: Formalin-fixed paraffin-embedded allograft kidney biopsies with acute cellular rejection $(\mathrm{ACR})(\mathrm{n}=7)$ and chronic antibody-mediated rejection (cAMR) $(n=8)$ were evaluated for IL-6 mRNA expression using RNAscope ${ }^{\circledR}$ in situ hybridization (ISH). The slides were co-stained with immunofluorescence (IF) using multiple tubular epithelial markers in order to localize ISH signals. Protocol biopsies taken 6 months post-transplant $(n=6)$ with no histologic abnormalities served as controls. The IL-6 ISH signal was quantitated from multiple representative fields of the cortex in the tubular and extra-tubular compartments using computer-assisted digital image analysis. Results: The total IL-6 ISH signal density was 216 and 234 points per $\mathrm{mm}^{2}$ in the renal cortex in the ACR and cAMR groups, respectively. The proportion of the extra-tubular signal was $67 \%$ in the ACR group and $75 \%$ in the cAMR group. The inflammatory cells appeared to be the primary source of the extra-tubular IL-6 ISH signal in the ACR group. 
In the cAMR group, inflammatory cells, endothelial cells, and some vascular smooth muscle cells were positive for IL-6 ISH signal in the extra-tubular compartment. None of the control protocol biopsies revealed any detectable IL-6 signal.

Conclusions: This study, using a highly sensitive and specific ISH combined with multiplex IF and computer-assisted digital image analysis quantitatively identifies the cellular source of intragraft IL-6 mRNA expression in kidneys with ACR and cAMR. Intragraft IL-6 mRNA expression by non-resident inflammatory cells and various resident cells in the kidney may promote rejection and tissue injury and as such, may provide a potential therapeutic target in both cell-mediated and antibody-mediated rejection.

\section{Impact of Insufficient Renal Biopsy Specimens}

JL Daghe, LA Schildmeier, CL Phillips. Indiana University, Indianapolis, IN; Park Avenue Solutions, Fishers, IN.

Background: Percutaneous needle biopsy of the kidney is the preferred specimen procurement method for diagnosing medical renal pathology. Although biopsy cores are relatively small, their acquisition carries risk to patients (vasovagal reaction, pain, bleeding, hematoma, infection, and rarely death), and their processing and interpretation consumes disproportionately greater technical time and reagents due to reliance on 3 modes of analysis requiring diverse processing techniques: light microscopy (LM), immunofluorescence (IF) and electron microscopy (EM). Successful microscopic interpretation of glomerulonephritis is dependent on quantity and quality of sampled glomeruli. Inadequate specimens with insufficient tissue, especially diminished glomeruli, place added burden on labs if tissue requires additional sectioning or crossprocessing among the 3 modes. Importantly, patient risk is doubled if repeat biopsy is performed.

Design: We retrospectively searched over 3000 computer archived reports corresponding to renal biopsy specimens processed over 42 months in a large hospital system. Insufficient samples were identified by SNOMED codes and the appearance of "insufficient" in the report. Flagged cases were analyzed for number of glomeruli present for LM and EM, where specimen adequacy was defined as containing a minimum of one patent glomerulus for EM and 14 glomeruli for LM (Wang, Nephrol Dial Transplant, 1998). Process mapping was utilized to determine time and path for processing EM samples.

Results: We identified 319 cases coded as insufficient, of which $96(30 \%)$ had $<14$ glomeruli for LM. In many of these cases, attempts were made to salvage tissue for additional histopathology by collection of deeper paraffin levels, or cross-processing tissue (e.g., IF tissue to LM, or LM to EM). For EM, processing of the initial specimen required on average 8 hours of technician effort from the time the specimen was received to capture of digital ultramicrographs. When EM tissue was insufficient for diagnosis, sample reprocessing required at least 5 additional hours of technician effort. In one 12-month period, $110 \mathrm{EM}$ cases proved to be insufficient due to lack of diagnostically useful glomeruli, with over 550 hours of additional technician effort (nearly 14 weekdays)

Conclusions: Inadequate renal biopsy specimens were identified in more than $10 \%$ of cases. Reprocessing of biopsy specimens may result in new diagnostic information that averts the need for repeat biopsy, but the additional workload places significant financial burdens and time constraints on labs and delays rendition of a final diagnosis.

$1674 \quad$ Interstitial Eosinophilic Aggregates in Diabetic Nephropathy Are Associated with the Severity of Chronic Tubulointerstitial Injury but Not with History of Medication Use or Allergy

$D-F$ Dai, K Sasaki, C Alpers, B Najafian. University of Washington, Seattle, WA.

Background: Although an interstitial eosinophilic aggregate (IEA) in renal biopsies is often considered a finding suggestive of an allergic type tubulointerstitial nephritis (TIN), it is not uncommon that an obvious correlation with drug allergy is not identified. IEA is commonly encountered in diabetic nephropathy (DN) and thought to be related to chronic medication use.

Design: Native kidney biopsies performed between 2000 and 2011 at UWMC were reviewed, including 64 cases of DN and 111 cases of non-DN cases, which were further classified as $\operatorname{IgA}$ nephropathy ( $\operatorname{IgAN}, \mathrm{n}=28)$, membranous nephropathy ( $\mathrm{MN}, \mathrm{n}=14)$, focal and segmental glomerulosclerosis (FSGS, $n=27$ ) and membranoproliferative glomerulonephritis (MPGN, $n=28$ ). IEA was defined as the presence of $\geq 5$ interstitial eosinophils /HPF. The severity of chronic tubulointersitial injury (interstitial fibrosis and tubular atrophy, IFTA) was scored from 0-3, i.e. minimal, mild, moderate and severe. Patient's demography, history of drug allergy and medication use within 2 months prior to biopsy was obtained from electronic medical records.

Results: IEA was significantly more prevalent in DN (41\%), when compared with IgAN $(7 \%, \mathrm{p}=0.001), \mathrm{MN}(8 \%, \mathrm{p}=0.017)$, or MPGN $(14 \%, \mathrm{P}=0.013)$, while the difference with FSGS $(26 \%)$ was not statistically significant $(\mathrm{p}=0.18)$. IEA in $\mathrm{DN}$ was predominantly found in the cortex and in association with IFTA. In DN cases, univariate analysis revealed that IEA was significantly associated with greater IFTA score, but not with the $\%$ of glomerulosclerosis, mesangial expansion, history of drug allergy, number of prescribed medications, or particular class of medications (antibiotics, NSAIDs, aspirin, thiazide, loop diuretics, ACEI, angiotensin receptor blockers, calcium channel blocker, beta blocker, insulin, sulfonylurea, metformin, or allopurinol). Multivariate analysis showed IFTA score was the only significant predictor $(\mathrm{p}<0.01)$ after stepwise adjustment for age, number of medication, history of allergy, type of diabetes, $\%$ of global glomerulosclerosis and mesangial expansion.

Conclusions: Our study shows that IEA is more common in DN, when compared with other types of glomerulopathy, and that in DN, IEA is associated with chronic tubulointerstitial injury (IFTA) but not with prescribed medications or clinical history of allergy. Our observation suggests that IEA may be related to chronic tubulointerstitial injury in the setting of DN and may not necessarily reflect an allergic type interstitial nephritis.

1675 Glomerular Microvessel Quantitation in Human Renal Biopsies Using Digital Image Analysis Demonstrates Alterations in Disease

CL Ellis, AB Farris, TE Rogers, D Lawson, C Cohen, S Rosen. Emory University, Atlanta, GA; Beth Israel Deaconess Medical Center, Harvard University, Boston, MA. Background: Glomeruli are vascular structures that undergo architectural remodeling with disease $(\mathrm{Dz})$ that is readily appreciable in a qualitative manner in routine nephropathology practice; however, objective, quantitative methods are not routinely employed. Glomerular microvascularity was quantitated in an array of Dz using image analysis to understand baseline architecture as well as fundamental structural changes. Design: A slide scanner was used to obtain whole slide images (WSIs) of native renal biopsies $(\mathrm{n}=44)$. Microvascularity was highlighted with CD34 immunohistochemistry. Glomeruli ( $\mathrm{n}=711$ ) were circumscribed, and microvascular measurements were performed with a microvessel image analysis algorithm.

Results: Glomeruli had a mean microvessel density (MVD) [in $10^{-4}$ vessels $/ \mathrm{um}^{2}$ ] of 14 (standard error $[\mathrm{SE}]=0.36$ ), which was significantly higher than the average tubulointerstitial MVD (mean $\pm \mathrm{SE}=1.8 \pm 0.3$, t-test $\mathrm{p}<0.0001)$. Furthermore, glomerular MVD had a wide range (1.2-40). Completely sclerotic glomeruli were excluded since MVD was essentially 0. Glomerular vessels had a mean area of $74 \mathrm{um}^{2}\left(\mathrm{SE}=7.5 \mathrm{um}^{2}\right)$, perimeter of $56.3 \mathrm{um}(\mathrm{SE}=3.4 \mathrm{um})$, and wall thickness of $1.2 \mathrm{um}(\mathrm{SE}=0.04 \mathrm{um})$. Mean glomerular profile area was $20,657 \mathrm{um}^{2}\left(\mathrm{SE}=430 \mathrm{um}^{2}\right)$. As shown in the figure, a statistically significant difference in MVD (t-test $\mathrm{p}<0.0001)$ was seen when glomeruli were compared on the basis of nonocclusive glomerular capillary loop Dz (normal, acute tubular injury [ATI], chronic interstitial nephritis [CIN], and membranous glomerulonephritis [MGN]) and occlusive capillary loop Dz (diabetes [DM], focal segmental glomerulosclerosis [FSGS], GS not otherwise specified [GS,NOS], IgA nephropathy, lupus [SLE], light chain deposition Dz [LCDD], membranoproliferative GN [MPGN], and monoclonal immunoglobulin deposition Dz [MIDD]) with a higher mean MVD in nonocclusive Dz compared to occlusive Dz.

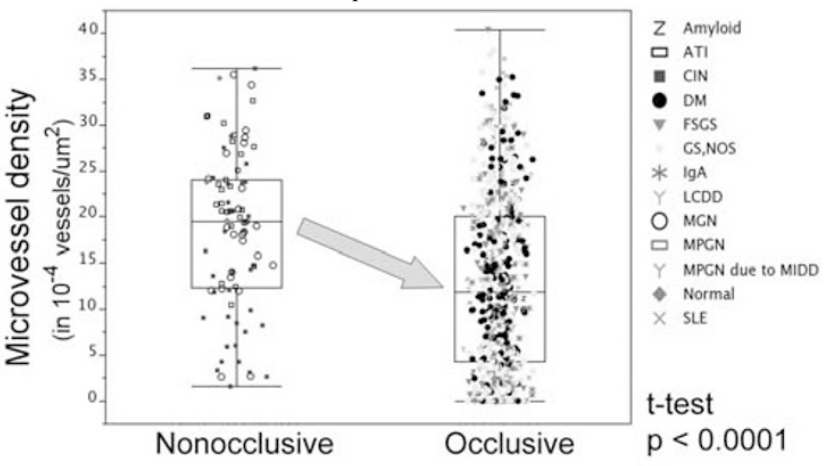

Conclusions: As expected, glomeruli are highly vascular structures that undergo a spectrum of microvessel architectural changes with Dz; however, there is Dz heterogeneity, indicating that further study may be worthwhile in larger cohorts and a wider variety of diseases.

\section{Establishing a Grading System of PAS Staining for Evaluation} of Acute Tubular Injury

$M$ Eskandari, W Li, MT Rooney, PL Zhang. William Beaumont Health System, Royal Oak, MI.

Background: Periodic Acid Schiff (PAS) is a stain routinely used in renal biopsies to identify acute tubular injury. Thus far, a standardized correlation between the degree of acute tubular injury (ATI) on PAS stained sections and renal dysfunction has not been established. In this study, we sought to determine PAS scoring system for ATI. Design: 25 renal biopsies from various renal diseases were randomly selected and evaluated for the degree of renal tubular brush border PAS staining as follows: [0] for intact brush borders (no injury), $[1+]$ for scattered loss and minimally diminished brush border (mild injury), $[2+]$ for diffuse loss with moderately diminished brush border (moderate injury), and [3+] for total loss of brush border (severe injury). In addition, all biopsies were stained for KIM-1 and graded from 0 to $3+$, based on their staining intensity. PAS scores, KIM-1 scores, and $\mathrm{sCr}$ levels were statistically evaluated by linear regression analysis.

Results: Ages ranged from 15 to 84 years old ( 12 male and 13 female). The sCr levels ranged from 0.63 to $15.3 \mathrm{mg} / \mathrm{dl}($ mean $=4.55)$. KIM-1 scores (arbitrary units on X-axis) showed significant linear correlation with $\mathrm{sCr}$ (Y-axis) $(\mathrm{R}=0.632, \mathrm{P}=0.0007)$ and the odds ratio between the two indices was $4.543(95 \%$ confidence interval $(\mathrm{CI})=$ $3.381-5.733$ ), implying that for every unit of increased KIM-1 score, the odds of $\mathrm{sCr}$ increased by $4.543 \mathrm{mg} / \mathrm{dl}$. Similarly, PAS scores (arbitrary units on X-axis) had statistically significant linear correlation with $\mathrm{sCr}$ (Y-axis) $(\mathrm{R}=0.543, \mathrm{P}=0.0051)$ and the odds ratio was $4.536(95 \% \mathrm{CI}=3.282-5.832)$. There was also a significant linear correlation between KIM-1 score and PAS score $(\mathrm{R}=0.780, \mathrm{P}=0.0001)$

Conclusions: The association of PAS scores with the $\mathrm{sCr}$ levels paralleled the association of KIM-1 scores with the sCr levels. Thus, we concluded that the current grading system of the PAS staining, developed in this study, can be a useful tool to confirm the degree of ATI. 
1677 Medullary and Cortical Microvascularity Show Parallels through Computerized Morphometic Analysis in Human Renal Biopsies

AB Farris, CL Ellis, TE Rogers, D Lawson, C Cohen, S Rosen. Emory University Hospital, Atlanta, GA; Beth Israel Deaconess Medical Center, Harvard University, Boston, MA

Background: The assessment of renal tubulointerstitial and vascular injury is important for both native and transplant kidneys; however, past studies have primarily focused on the renal cortex. Microvascular anatomic features were quantitated in both the renal cortex and medulla in order to investigate the utility of image analysis in this setting as well as to determine relationships between cortex and medulla.

Design: Consecutive native renal biopsies $(n=44)$ with medullary tissue available for evaluation were included for analysis. Tubulointerstitial microvascularity was highlighted with CD34 immunohistochemistry. Whole slide images were digitally scanned. Microvascularity was quantitated with a microvessel algorithm, which provided a measure of microvessel density (MVD), mean vascular area (MVA), perimeter, and wall thickness.

Results: The renal biopsies had heterogeneous disease, ranging from an essentially normal biopsy in which the MVD (in $10^{-5}$ vessels $/ \mathrm{um}^{2}$ ) was 80 in all of the tissue (cortex + medulla), diminishing to 2.8 in the setting of extensive interstitial fibrosis and tubular atrophy. The mean MVD for all tissue was 18 (standard error $(\mathrm{SE})=2.9$ ). MVA for all tissue ranged from $42-127 \mathrm{um}^{2}$ (mean $\left.\pm \mathrm{SE}=82 \pm 3.7\right)$. As shown in the figure, there were correlations among MVD and MVA in the cortex, medulla, and entire parenchyma taken as a whole with the tightest correlation seen between the medulla and entire tissue $(r=$ $0.91, \mathrm{p}<0.001$ for MVA); however, histograms depict a wide range in the measured parameters. Other parameters correlated among the compartments, including vascular perimeter (all tissue mean $\pm \mathrm{SE}=56 \pm 1.4 \mathrm{um} ; \mathrm{r}=0.50-0.67$ between compartments, $\mathrm{p}$ $=<0.0001$ to 0.0006 ) and thickness (all tissue mean $\pm \mathrm{SE}=1.4 \pm 0.04 \mathrm{um} ; \mathrm{r}=0.86-0.91$, $\mathrm{p}<0.0001$ for all comparisons)

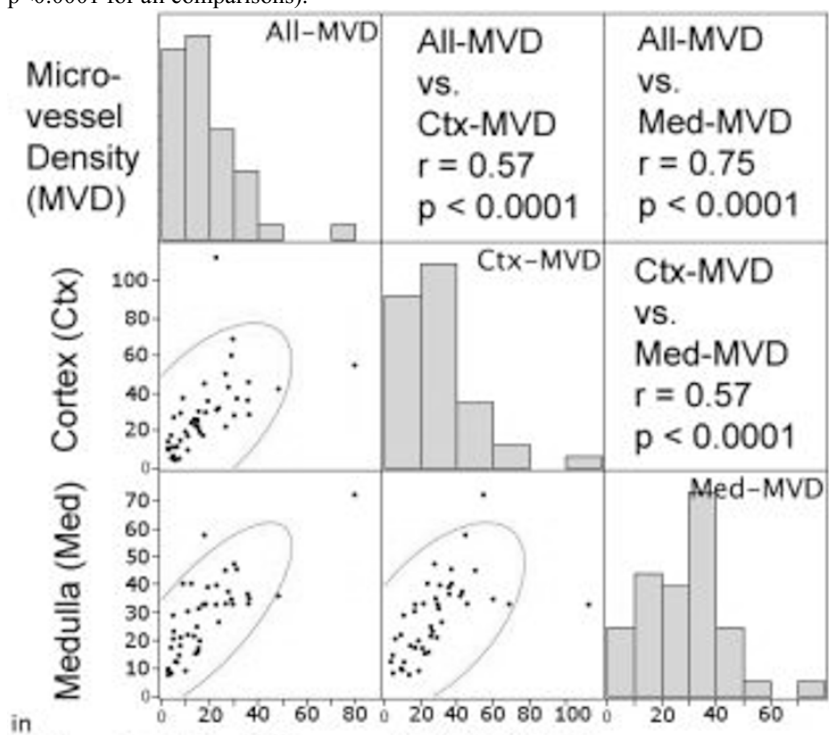

$10^{-5}$ vessels $/ \mathrm{um}^{2}$ All Cortex $(\mathrm{Ctx})$

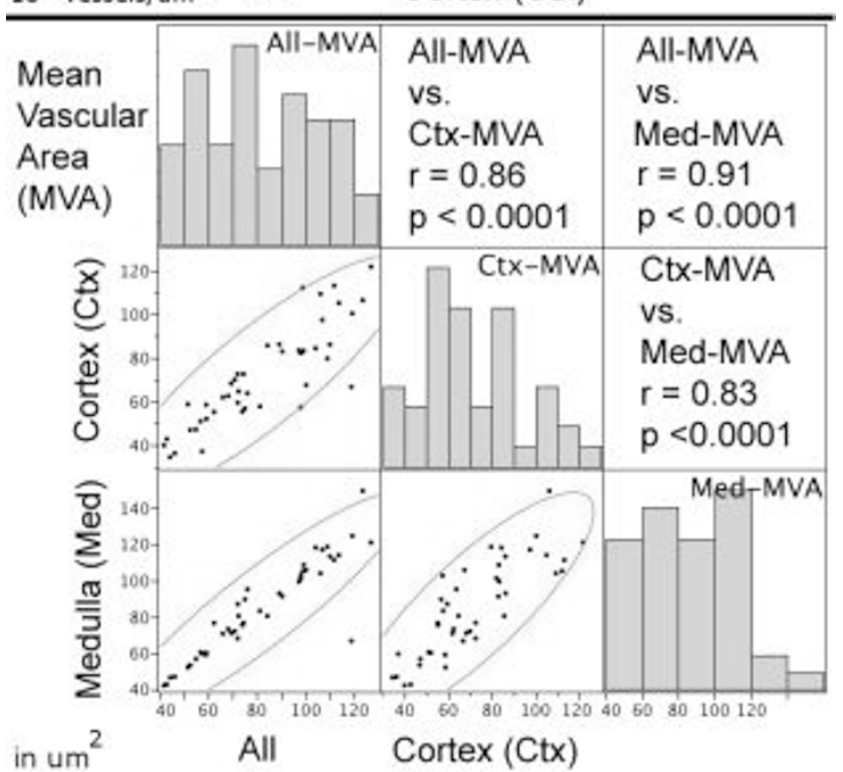

Conclusions: Renal medullary vascularity shows parallels with the renal cortex and the entire renal parenchyma as a whole with regard to parameters such as MVD, MVA, vascular perimeter, and thickness. However, there is wide heterogeneity, indicating that further study may be worthwhile in larger cohorts and a wider variety of diseases.
1678 Mesangial Immune Complex Deposits in Membranous Glomerulonephritis Mediated by Anti-Phospholipase A2 Receptor Autoantibodies

AM Gasim, LH Rivier, K Dittrich, P Nachman, JC Jennette. University of North Carolina at Chapel Hill, Chapel Hill, NC.

Background: Current concepts of membranous glomerulonephritis (MGN) suggest that mesangial immune complex deposits (MD) increase the likelihood of "secondary" MGN caused by a systemic source for antigens rather than primary MGN with complexes containing podocyte antigens present in the subepithelial zone. e.g. phospholipase A2 receptor (PLA2R). Based on these concepts, MGN with no mesangial deposits should contain PLA2R more often than MGN cases with mesangial deposits (MGN MD).

Design: We selected $34 \mathrm{MGN}$ cases with no clinical evidence for secondary MGN, including no malignancy, and negative ANA, anti-dsDNA, HBV, HCV, HIV, and RPR. For comparison, we selected 7 specimens with lupus MGN with no proliferative features. 22 of 34 cases with non-lupus (NL) MGN were selected with no mesangial deposits (NL MGN NMD), and 12 with mesangial deposits (NLMGN MD). All specimens were stained for PLA2R1 using immunofluorescence microscopy on FFPE tissue (anti-PLA2R from Sigma-Aldrich). Immunoglobulin and complement staining was performed using standard immunofluorescence techniques on frozen sections. Results: As expected, SLE MGN was negative for PLA2R, and had demographic and pathologic features that differed from those of NL MGN. Unexpectedly, most NL MGN with mesangial deposits were PLA2R+. The immunoglobulin composition of deposits differed between NL MGN with and without mesangial deposits.

Table 1

\begin{tabular}{|c|c|c|c|c|c|c|c|}
\hline & n & $\begin{array}{l}\text { Age (Mean/ } \\
\text { range) }\end{array}$ & $\begin{array}{l}\text { Sex } \\
(\mathrm{M} / \mathrm{F})\end{array}$ & $\begin{array}{l}\text { Race }(\mathrm{B} / \mathrm{W} / \\
\text { other) }\end{array}$ & $\begin{array}{l}\% \\
\text { PLA2R+ }\end{array}$ & \begin{tabular}{|l} 
Mean \\
PLA2R \\
$0-4+*$
\end{tabular} & TBM Deposit \\
\hline $\begin{array}{l}\text { NL MGN } \\
\text { NMD }\end{array}$ & 22 & $55 / 7-77$ & $13 / 9$ & $\mid 14 / 4 / 4$ & $77 \%$ & $2.5+$ & $0 \%$ \\
\hline $\begin{array}{l}\text { NL MGN } \\
\text { MD }\end{array}$ & 12 & $63 / 24-80$ & $7 / 5$ & $8 / 2 / 2$ & $67 \%$ & $2.4+$ & $0 \%$ \\
\hline \begin{tabular}{|l} 
SLE \\
MGN
\end{tabular} & 7 & |37/20-46 & $1 / 6$ & $2 / 5 / 0$ & $0 \%$ & NA & $86 \%$ \\
\hline
\end{tabular}

*mean intensity when positive

\begin{tabular}{|c|c|c|c|c|c|c|c|}
\hline & $n$ & $\begin{array}{l}\%+\operatorname{IgG} 1 / \operatorname{lgG} 2 / \\
\operatorname{lgG} 3 / \mathrm{IgG} 4 *\end{array}$ & $\begin{array}{l}\text { Mean 0-4+IgG1/ } \\
\mathrm{IgG} 2 / \mathrm{IgG} 3 / \operatorname{IgG} 4 *\end{array}$ & IgA* & IgM* & C3* & Clq* \\
\hline $\begin{array}{l}\mathrm{NL} \\
\text { MGN } \\
\text { NMD }\end{array}$ & 20 & $100 / 100 / 100 / 95$ & $2.9 / 2.0 / 1.8 / 3.1$ & $45 \% / 1.1+$ & $68 \% / 0.76+$ & $95 \% / 1.7+$ & $54 \% / 0.7+$ \\
\hline \begin{tabular}{|l} 
NL \\
MGN \\
MD
\end{tabular} & 9 & $100 / 89 / 100 / 78$ & $3.1 / 1.9 / 2.5 / 2.6$ & $83 \% / 0.85+$ & $83 \% / 0.85+$ & $100 \% / 1.6+$ & $67 \% / 0.8+$ \\
\hline $\begin{array}{l}\text { SLE } \\
\text { MGN }\end{array}$ & 7 & $\mid 100 / 100 / 100 / 100$ & $3.3 / 3.4 / 2.5 / 3.2$ & $100 \% / 1.2+$ & $100 \% / 1.0+$ & $100 \% / 2.5+$ & $86 \% / 2.3+$ \\
\hline
\end{tabular}

$* \%$ positve and mean intensity when positive $(0-4+)$

Conclusions: Mesangial immune complex deposits in MGN do not rule out PLA2R mediated MGN; in fact, most NL MGN with mesangial deposits apparently is caused by anti-PLA2R. In accord with other studies, PLA2R in immune deposits is not frequent in SLE MGN. Hypothetically, the PLA2R in mesangial deposits could derive from diffusion from paramesangial podocytes into the mesangial matrix, or from a circulating source for PLA2R.

1679 Claudin-1 and Nephrin Label Cellular Crescents in Diabetic Glomerulosclerosis

JP Gaut, M Hoshi, S Jain, H Liapis. Washington University School of Medicine, St. Louis, MO.

Background: Cellular crescents are typically inflammatory and associated with rapidly progressive glomerulonephritis. Their pathogenesis involves glomerular basement membrane rupture due to circulating or intrinsic factors. Crescents associated with diabetic glomerulosclerosis are rarely reported. Furthermore, the nature of cells forming crescents in diabetes is unknown.

Design: To investigate the nature of crescents in diabetes, we used renal biopsies from diabetic patients with nodular glomerulosclerosis and crescents $(\mathrm{n}=2)$, diabetes without crescents $(n=5)$, non-diabetic renal biopsies $(n=3)$ and crescentic glomerulonephritis with inflammatory crescents from patients with vasculitis as controls $(n=3)$. Electron microscopy and immunofluorescence analysis with antibodies against nephrin (a podocyte marker) and claudin-1 (parietal epithelial cell marker) were performed.

Results: Diabetic glomeruli with crescents showed the majority of crescentic cells expressing either marker exclusively. Rare crescentic cells co-expressed nephrin and claudin-1 compared to controls. Cells co-expressing claudin-1 and nephrin were absent in the controls. Electron microscopy showed podocyte bridge formation between the glomerular basement membrane and parietal basement membrane but no GBM rupture as in inflammatory crescents.

Conclusions: Crescents in diabetes may occur in the absence of a secondary etiology and are composed of a mixture of parietal epithelial cells and visceral podocytes. Cells co-expressing parietal epithelial and podocyte markers suggest that parietal epithelial cells may transdifferentiate into podocytes in response to severe glomerular injury.

1680 Inflammatory Reaction Is Necessary for B-Catenin Nuclear Translocation in the Fibrosed Interstitial Areas in Diabetic Nephropathy $X \mathrm{Gu}$, GA Herrera. LSUHSC-Shreveport, Shreveport, LA.

Background: Diabetic nephropathy (DN) is a major complication of diabetes and a frequent cause of end-stage renal disease. Recent studies indicate that Win/B-catenin pathway is involved in high concentration glucose-induced cellular injury in the kidney. In the interstitium, activation of Win/B-catenin pathway is also involved in 
epithelial mesenchymal transition and may responsible for interstitial fibrosis. However, information of B-catenin expression in human DN is limited. We have studied B-catenin expression and nuclear translocation, a morphologic indication of Win pathway activation, in DN kidney biopsies.

Design: Twenty kidney biopsies with DN were selected from archived material. Fourmicron sections were prepared. B-catenin immunohistochemical stains were performed following standard laboratory protocols.

Results: All cases showed nodular glomerulosclerosis or significant expansion of mesangium. The glomerular podocytes were negative for B-catenin. Endothelial cells, mesangial cells and parietal epithelial cells revealed cytoplasm expression for B-catenin. Tubular epithelial cells and vascular endothelial cells also showed strong cytoplasm expression for B-catenin. Sixteen of 20 cases revealed focal interstitial fibrosis. Positive nuclear staining was identified in scattered tubular cells, vascular endothelial cells, fibroblastic stromal cells and inflammatory mononuclear cells. The nuclear positive cells are only present in the fibrosed interstitial areas that showed inflammatory infiltrates. In the fibrosed areas lacking inflammatory cells, no positive nuclear reaction was identified.

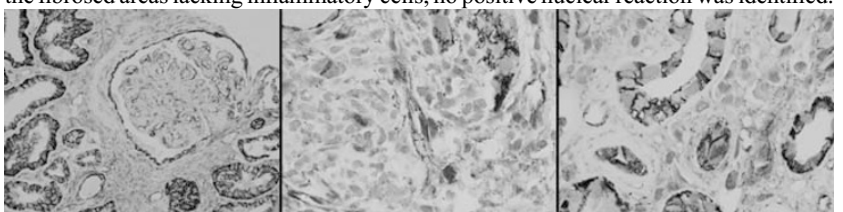

Left: Positive cytoplasm stain in glomerular endothelial, mesangial, parietal cells and tubular epithelial cells. Middle: Positive nuclear staining in capillary endothelial cells and stromal fibroblasts in a background of mononuclear inflammatory cell infiltration. Right: Positive nuclear staining in tubular epithelial cells with adjacent mononuclear inflammatory cells

Conclusions: Nuclear translocation is limited to the fibrosed interstitial areas with inflammatory reaction. The finding suggests that presence of proinflammatory cytokines is necessary for B-catenin translocation in interstitial fibrosis in $\mathrm{DN}$.

\section{Amyloidogenic Light Chain Cast Nephropathy}

V Kaushik, L Cornell, M Fidler, S Sethi, L Herrera Hernandez, M Alexander, J Grande, G Phadke, J Shanks, S Nasr. Mayo Clinic, Rochester, MN; Sanford Health, Fargo, ND; St. John's Hospital, Maplewood, MN.

Background: Light chain cast nephropathy (LCCN) is the most common finding in multiple myeloma (MM) patients (pts) with acute renal failure. Typical light chain (LC) casts appear fractured and stain hypereosinophilic on H\&E, and PAS and Congo red-negative. Rarely, LC casts are composed of amyloid (amyloidogenic (ALCCN). There have been no series addressing the characteristics of ALCCN.

Design: We identified 17 cases of ALCCN by a retrospective review of our Pathology archives from 1994-2013. All cases showed LC casts that were Congo red-positive with apple-green birefringence under polarized light.

Results: The mean pt age was 60 yrs (range, 41-72), with a M:F of 1.1. All 13 pts with available data had MM, which was diagnosed concurrently with ALCCN in most pts. Bone marrow biopsy showed LC-restricted plasma cells in all (mean $44 \%$, range $5-95 \%)$, but was positive for amyloid in only $1(8 \%)$. The latter pt also had amyloid in the left knee joint. One pt had hepatic encephalopathy which was suspected to be due to amyloidosis involvement but he expired before histological confirmation. None of the other pts had clinical or histologic evidence of extrarenal amyloidosis. Anemia, lytic lesions, and hypercalcemia were present in $100 \%, 58 \%, 27 \%$ of pts, respectively. The monoclonal protein on S. immunofixation, available on 15 , was $\lambda$ in $13, \kappa$ in 1 , and IgG without LC in 1 who had heavy chain disease. Serum free light chain ratio, tested in 12 , was markedly abnormal in 11 and normal in 1 . All pts presented with acute renal failure with a mean S. creatinine of $6.5 \mathrm{mg} / \mathrm{dl}$. The median $24 \mathrm{~h}$ urine protein was 5.1 $\mathrm{g}$, and most of the urinary protein was composed of LC. Edema was present in $8 \%$ and hematuria in $18 \%$, but none had nephrotic syndrome. Pts were treated with chemotherapy with/without stem cell transplant. After a mean F/U of 14 mos (range 1-42), available in $12 \mathrm{pts}, 33 \%$ progressed to ESRD. Hematologic response occurred in $63 \%$ of pts, while $25 \%$ died of myeloma complications. Histologically, the amyloidogenic casts appeared pale on H\&E and PAS-negative. $87 \%$ of cases showed spicules at the periphery of casts. The pt with confirmed extrarenal amyloidosis also had amyloid within tubular cells. No case showed amyloid in glomeruli or vessels. All cases except 1 revealed LC chain restriction on immunofluorescence ( $\lambda$ in $15, \kappa$ in 1$)$.

Conclusions: ALCCN is a distinct morphologic variant of LCCN and is usually of the $\lambda$ type. Pts clinical characteristics and prognosis appear comparable to classic LCCN. The vast majority of pts do not have concurrent glomerular, vascular, or extrarenal amyloidosis.

1682 Correlation of Renal Structural Pathologic Features (Microanatomy) with Radiologic Features (Macro-Anatomy) in Living Kidney Donors

V Kaushik, AD Rule, H Elsherbiny, LD Cornell, MP Alexander. Mayo Clinic College of Medicine, Rochester, MN.

Background: While renal pathology has been characterized in patients with clinically evident kidney disease, the characteristics of renal pathology in normal adults is less well defined. Our objective was to determine if contrast computed tomography (macroanatomy) could detect structural pathology (micro-anatomy) on renal biopsy.

Design: Living kidney donors (2000-2011) had contrast computed tomographic angiograms just prior to kidney donation with an implantation renal biopsy. Macroanatomy was characterized by cortical volume (V), medullary V, cysts, surface roughness, renal artery atherosclerosis. Micro-anatomy was characterized by nephron size (glomerular V, cortical area per glomerulus, and profile tubular area) and nephrosclerosis (\% ischemic or sclerotic gloms, $\%$ interstital fibrosis, $\%$ intimal occlusion, and arteriolar hyalinosis). Spearman's rank correlation was performed.

Results: There were 1039 donors (female 59\%, mean age 44 yrs) studied. Figure 1 summarizes the macro-microanatomy correlations $(*$ for $\mathrm{p}<0.05$ ). Total cortex volume or cortex volume/medulla volume correlate positively with measures of increased nephron size, which are non sclerotic glomerular volume(NSGV), mean tubular profile area, cortical area per glomerulus and negatively with interstitial fibrosis,percentage of ischemic/sclerotic glomeruli and intimal thickness on biopsy. Decreased cortico/ medullary ratio, presence of cysts, and renal artery atherosclerosis correlate positively with measures of nephrosclerosis on renal biopsy.

Conclusions: Among healthy adults, contrast computed tomography can detect underlying variation in nephron size and specifically nephrosclerosis.

\begin{tabular}{|c|c|c|c|c|c|c|c|}
\hline & \multicolumn{3}{|c|}{ Nephr } & \multicolumn{2}{|c|}{ Nephrosclerosis } & & \\
\hline & $\begin{array}{l}\begin{array}{l}\text { Glom } \\
\text { rolume }\end{array} \\
\end{array}$ & \begin{tabular}{|l} 
Cortex \\
area/Glom
\end{tabular} & $\begin{array}{l}\text { Tubular } \\
\text { profile } \\
\text { area }\end{array}$ & $\begin{array}{l}\% \text { of } \\
\text { Ischemic/S } \\
\text { clerotic } \\
\text { glomeruli }\end{array}$ & \% Fibrosis & Hyalinosis & $\begin{array}{l}\text { Intimal } \\
\text { thickness }\end{array}$ \\
\hline Cortex Vol & $0.27 *$ & $0.13^{\star}$ & $0.17^{\star}$ & $-0.09^{\star}$ & $-0.07^{\star}$ & 0.02 & $-0.06^{\star}$ \\
\hline $\begin{array}{l}\text { Cortex / } \\
\text { medulla } \\
\text { (rol) }\end{array}$ & $0.19^{*}$ & $0.10^{\star}$ & $0.11^{*}$ & $-0.12^{*}$ & $-0.09^{*}$ & -0.02 & $-0.15^{*}$ \\
\hline Roughness & -0.00 & 0.05 & 0.04 & $0.06^{*}$ & $0.11 *$ & $0.06^{*}$ & $0.10^{*}$ \\
\hline Cysts & 0.01 & 0.04 & 0.03 & $0.11^{*}$ & $0.07^{*}$ & 0.01 & $0.07^{*}$ \\
\hline $\begin{array}{l}\text { Arterial } \\
\text { sclerosis }\end{array}$ & -0.01 & 0.05 & 0.03 & $0.10^{*}$ & 0.09 * & $0.10^{*}$ & 0.05 \\
\hline
\end{tabular}

1683 Post-Infectious Glomerulonephritis in the Era of C3 Glomerulopathy: A New Look at an Old Disease

MA Khalighi, S Meehan, A Chang. University of Chicago, Chicago, IL.

Background: $\mathrm{C} 3$ glomerulonephritis $(\mathrm{C} 3 \mathrm{GN})$ is a new entity, which is defined by C3 dominant immunofluorescence (IF) staining. Recently, a subset of post-infectious glomerulonephritis (PIGN) with atypical features was found to have abnormalities in the alternative complement pathway similar to $\mathrm{C} 3 \mathrm{GN}$. Therefore, we applied the current diagnostic criteria for $\mathrm{C} 3 \mathrm{GN}$ to our cases of PIGN to determine the implications on our clinical practice.

Design: We identified 54 biopsies diagnosed as PIGN from 2005-2013, and using recent diagnostic criteria for $\mathrm{C} 3 \mathrm{GN}$ we divided them into two groups: 1) 0 to $1+\mathrm{IF}$ staining (using a $0-4+$ scale) for immunoglobulins (Ig) and 2) $2+$ or greater staining for Ig. C 3 staining was at least $2+$ in all cases and at least $3+$ in all cases with $1+$ Ig staining. Histopathologic features were reviewed and compared between the two groups. Results: Of 54 kidney biopsies with a diagnosis of PIGN, 27 (16 adults, 11 children) showed dominant $\mathrm{C} 3$ staining by IF with 0 to $1+$ staining for Ig and 27 (17 adults, 10 children) demonstrated $2+$ or greater staining for $\operatorname{IgG}(n=21)$ or $\operatorname{IgA}(n=6)$. The serum creatinine at diagnosis was similar in both groups (mean of $4.3 \mathrm{mg} / \mathrm{dL}$ (range: 1.4-10) in the $0-1+$ group and $4.1 \mathrm{mg} / \mathrm{dL}$ (range: $1-14$ ) in the $2+$ or greater group).

\begin{tabular}{|l||l|l||l||}
\hline Pathologic Features & C3 Dominant $(\mathrm{n}=27)$ & C3 Co-Dominant $(\mathrm{n}=27)$ & $\mathrm{P}$ value \\
\hline Exudative GN & $21(78 \%)$ & $25(93 \%)$ & $\mathrm{p}=0.12$ \\
\hline Crescents & $12(44 \%)$ & $7(26 \%)$ & $\mathrm{p}=0.13$ \\
\hline Garland IF & $4(15 \%)$ & $12(48 \%)(\mathrm{n}=25)$ & $\mathrm{p}=0.01^{*}$ \\
\hline Starry sky IF & $21(78 \%)$ & $12(48 \%)(\mathrm{n}=25)$ & $\mathrm{p}=0.03^{*}$ \\
\hline Mesangial IF & $2(7 \%)$ & $1(4 \%)(\mathrm{n}=25)$ & $\mathrm{p}=0.53$ \\
\hline Subepithelial Hump Deposits & $25(93 \%)$ & $25(93 \%)$ & $\mathrm{p}=0.69$ \\
\hline Intramembranous Deposits & $25(93 \%)$ & $15(56 \%)$ & $\mathrm{p}=0.002^{*}$ \\
\hline
\end{tabular}

* statistically significant

Conclusions: Half of the PIGN cases at our institution would now be diagnosed as C3GN based on the new criteria. These newly reclassified C3GN cases often demonstrated more crescents, starry-sky IF staining, and more commonly had intramembranous deposits by EM. Additional follow-up data and testing of the alternative pathway of complement may determine whether PIGN should be stratified into two subgroups or whether these cases should truly be reclassified as C3GN.

\section{Pediatric Pauci-Immune Glomerulonephritis}

MA Khalighi, A Chang, S Meehan. University of Chicago, Chicago, IL.

Background: Pauci-immune glomerulonephritis (GN) is the most common cause of crescentic GN in adults; however, it is much less common in children and pathologic features are less well described.

Design: We conducted a retrospective clinicopathologic study of 24 pediatric kidney biopsies with pauci-immune GN from 2005-2013. Available clinical data and histopathologic findings were reviewed and compared with 167 adult kidney biopsies diagnosed with pauci-immune GN

Results: Of 532 pediatric kidney biopsies in an eight year period, 24 biopsies (4.5\%) from 20 patients were diagnosed as pauci-immune GN. The average age was 13 years (range: 3-18). The female to male ratio was 2.3:1. The average serum creatinine at diagnosis was $3.5 \mathrm{mg} / \mathrm{dL}$ (range: $0.45-12$ ) in 14 patients with available data. Fifteen of 16 patients with available data were ANCA positive including P-ANCA $(31 \%, n=5)$ or C-ANCA $(13 \%, n=2)$ pattern without additional antigen specificity testing. Three $(19 \%)$ had anti-PR3 antibodies and $5(31 \%)$ had anti-MPO antibodies. One patient with unknown ANCA status was treated with propylthiouracil. Pauci-immune GN accounted for $18 \%$ of biopsies with crescents and was the third most common cause of crescents after lupus nephritis and IgA nephropathy. Active lesions (fibrinoid necrosis/cellular crescents) were identified in $18(75 \%)$ biopsies, while the remaining $6(25 \%)$ showed purely chronic lesions (fibrous crescents/global sclerosis). The average percentage of active necrosis and crescent formation was 51\% (range: 2-97\%). Based on the histologic classification system of Berden et al, crescentic GN was the most common pattern $(38 \%)$, followed by focal $(29 \%)$, sclerotic $(21 \%)$ and mixed $(13 \%)$. Of the 3 patients 
with follow-up biopsies, 2 progressed from crescentic to sclerotic $\mathrm{GN}$ and 1 progressed from mixed to sclerotic GN. Necrotizing arteritis was identified in 3 of 20 patients $(15 \%)$. In adults, the female to male ratio was $1.3: 1$. Histologically, 62 were crescentic $(37 \%), 68$ were focal $(41 \%), 19$ were sclerotic $(11 \%)$ and 18 were mixed $(11 \%)$. In patients with active fibrinoid necrosis or crescent formation, the mean percentage of necrosis or crescents was $43 \%$ (range: $3-100 \%$ ). 160 of the 167 biopsies had arteries for evaluation with $16(10 \%)$ showing necrotizing arteritis.

Conclusions: Children with pauci-immune glomerulonephritis showed a greater female predilection, and had a slightly higher rate of necrotizing arteritis than their adult counterparts. Histologically, the distribution of glomerular injury was similar in both groups.

1685 Histological and Clinical Evaluation of Adult Patients with HenochSchoenlein Purpura Nephritis from a Single Hospital in China: Review of 114 Cases

L Lai, H Li, M Azrad, JZhong, C-M Hao, JNovak, BA Julian, L Novak. Fudan University, Shanghai, China; University of Alabama at Birmingham, Birmingham, AL.

Background: Henoch-Schoenlein purpura nephritis (HSPN) has been more extensively studied in children than in adults. Manifestation of this disease in Chinese adult patients is not very well known. The aim of this study was to evaluate histopathological changes in renal biopsy tissue and to assess clinical data of a large cohort of 114 adult HSPN patients.

Design: Renal-biopsy slides were collected from 114 adult patients with $\operatorname{HSPN}(\mathrm{F}=63$, $\mathrm{M}=51$; age range $18-78 \mathrm{yr}$, average age $37 \mathrm{yr}$ ) who underwent biopsy from 2002 to 2013 . Two pathologists evaluated histopathological changes. Clinical data were compiled from review of medical records. To evaluate glomerular mesangial proliferation, cellularity of each glomerulus was graded (1-mild, 2-moderate, 3-severe) and a mean mesangial score was calculated for each biopsy, as described (Kidney International 76:54, 2009). In addition, 110 patients with known date of onset of purpura were grouped based on interval from the onset to renal biopsy: group $1(\mathrm{G} 1,<1$ month, $\mathrm{n}=14)$; group 2 (G2, $1-6$ months, $\mathrm{n}=58$ ) and group 3 (G3, $>6$ months, $\mathrm{n}=38$ ).

Results: In this cohort, all patients had purpura, proteinuria (average $2.07 \mathrm{~g} / 24 \mathrm{~h}$ ) and microscopic, but not macroscopic, hematuria. $4.4 \%$ patients had eGFR $[\mathrm{CG}]<50 \mathrm{~mL} /$ min, 27\% had abdominal pain and $26 \%$ had joint pain. Increased serum IgA level $(>3.9$ $\mathrm{g} / \mathrm{L}$ ) was detected in $18 \%$. G1 - G3 groups had similar mean 24-h proteinuria, hematuria (microscopic count of RBC in urinary sediment), mean eGFR and frequency of ACEI/ ARB treatment, but the percentage of blood neutrophils differed between the groups $(\mathrm{G} 1=71 \%, \mathrm{G} 2=66 \%, \mathrm{G} 3=57 \%, \mathrm{p}<0.001)$. Histopathology of the cohort showed mean mesangial score 1.1 (range $0.29-2.38$ ) and presence of segmental sclerosis (18\%), global sclerosis (26\%), glomerular crescents $(56 \%)$, glomerular adhesion $(26 \%)$, tubular atrophy $(43 \%)$, tubular casts $(46 \%)$, interstitial fibrosis $(39 \%)$, and presence of interstitial lymphocytes (51\%). Groups G1 - G3 did not differ in histopathology, except for median percentage of glomeruli with lymphocytes ( $\mathrm{G} 1=57 \%, \mathrm{G} 2=10 \%$, $\mathrm{G} 3=21 \%, \mathrm{p}<0.001)$ and mean percentage of the presence of interstitial fibrosis $(\mathrm{G} 1=$ $36 \%$, G2 $=31 \%$, G3 $=55 \%, \mathrm{p}=0.05$ ).

Conclusions: Patients biopsied in less than 1 month (G1) had higher percentage of glomerular lymphocytes and blood neutrophils. Severity of crescents was not related to the timing of biopsy after onset of purpura. This unique large cohort can serve for comparison with data on adult HSPN patients in other geographic locations.

\section{Leukocyte Chemotactic Factor 2 Amyloidosis (ALECT2): A Familial Amyloidosis?}

CP Larsen, ML Beggs, PD Walker. Nephropath, Little Rock, AR.

Background: Leukocyte chemotactic factor 2 amyloidosis (ALECT2) is a recently described form of amyloidosis that most frequently manifests clinically with progressive renal failure. We detail the largest case series of renal ALECT2 to date.

Design: We reviewed the case files of our institution for native kidney biopsy cases with amyloidosis. These biopsies were processed using routine light, IF, and EM. ALECT2 cases were identified using immunohistochemistry. The clinical and morphologic findings in each case with ALECT2 were reviewed. The LECT2 gene was sequenced in a subset of the cases using Sanger sequencing.

Results: ALECT2 was found to be the second most common form of renal amyloidosis in this series representing 40 of 414 total cases of renal amyloidosis $(9.6 \%)$. It is particularly common in Hispanic patients in the Southwest United States where greater than $50 \%$ of amyloidosis cases were LECT 2 type. We raise the possibility that this represents a familial amyloidosis as we discovered two brothers with ALECT2. Morphologically, there is consistent amyloid deposition in the renal cortical interstitium with medullary involvement in only about a third of cases. There were no mutations detected in the $L E C T 2$ gene though all patients were homozygous for the $\mathrm{G}$ nucleotide in a non-synonymous SNP at position 172 . Most patients presented with chronic kidney disease and, on follow-up, showed progression with an average deterioration in renal function of $0.5 \mathrm{~mL} / \mathrm{min} / 1.73 \mathrm{~m}^{2}$ per month.

Conclusions: ALECT2 is a newly described form of amyloidosis which frequently involves the kidney leading to renal failure. It is remarkably common considering the recent description of this disease and the prevalence is particularly high in patients of Hispanic ethnicity from the Southwest US. The etiology of ALECT2 is currently unknown though the over-representation of a common polymorphism in the LECT2 gene in patients with ALECT2 indicates that this change is necessary but not sufficient to cause disease. There is currently no treatment for this disease.
1687 Tubulointerstitial Injury Sensitizes to Glomerular Injury

BJ Lim, M-Z Zhang, RC Harris, H-C Yang, AB Fogo. Vanderbilt University Medical Center, Nashville, TN.

Background: It is well known that glomerular injury and loss of glomerular function can cause subsequent tubulointerstitial change via various mechanisms. However, little is known about the reverse process, i.e., spread of tubulointerstitial injury to glomerular injury. In this study, we evaluated the effect of preexisting tubulointerstitial injury on the glomerular function and the progression of glomerular scarring.

Design: By mating DT transgenic mouse, which expresses diphtheria toxin (DT) receptor in proximal tubular epithelial cells, with the Nep25 transgenic mouse which expresses human CD25 in podocyte, we generated Nep $25^{+} / D T^{+}(\mathrm{n}=4)$ and $N e p 25^{+} / D T$ mice. At day 0 and day 5 , all mice received DT injection $(100 \mathrm{mg} / \mathrm{kg}$, i.p). At week 6 , they underwent uninephrectomy to confirm the tubulointerstitial damage. Three days later, LMB2 $(8 \mathrm{ng} / \mathrm{g} \mathrm{BW}$, i.v.) was given to induce podocyte injury. All mice were sacrificed one week after LMB2 injection and the remaining kidneys were harvested. Results: After DT injection, there was more interstitial fibrosis in Nep $25^{+} / D T^{+}$mice than $N e p 25^{+} / D T$, assessed by Sirus red staining $\left(\right.$ Nep $25^{+} / D T^{+} 0.072 \pm 0.019 v$ vs. Nep $25^{+} /$ $D T 0.034 \pm 0.012 \%, \mathrm{P}<0.05)$. Before LMB2 injection, both groups showed normal range albuminuria (Urine albumin: creatinine ratio [ACR], Nep $25^{+} / D T^{+} 45.3 \pm 6.4 v$ s. Nep $25^{+} /$ DT $35.8 \pm 3.7 \mathrm{ug} / \mathrm{mg}$, pNS). Proteinuria increased more in Nep $25^{+} / D T^{+}$mice after LMB2 injection (Nep $25^{+} / D T^{+} 128.6 \pm 26.5,2.8$-fold $v$ s. baselline and Nep $25^{+} / D T$ 51.5 $535.5 \mathrm{ug} /$ $\mathrm{mg}, 1.4$-fold $v s$. baseline, $\mathrm{P}<0.05$ ). In parallel, there was more synaptopodin staining in Nep $25^{+} / D T^{+}$glomeruli than in Nep $25^{+} / D T$. Glomerulosclerosis was more widespread in Nep $25^{+} / D T^{+}$mice after LMB2 injection.

Conclusions: We conclude that preexisting tubulointerstitial injury made glomeruli more sensitive to glomerulus-specific injury. These results suggest that tubuloglomerular feedback is an important mechanism affecting the progression of glomerular injury.

1688 Quantitative Assessment of Density of Deposits in C3 Glomerulopathies

M Lusco, A Fogo. Vanderbilt University Medical Center, Nashville, TN.

Background: $\mathrm{C} 3$ glomerulopathy (C3GP) includes a range of diseases from $\mathrm{C} 3$ glomerulonephritis (C3GN) to dense deposit disease (DDD), all with $\mathrm{C} 3$ dominant deposits and an etiology of alternative complement pathway dysregulation. C3GN is distinguished from DDD by location and perceived density of deposits on electron microscopy (EM). In some cases, intermediate deposit density causes difficulty in distinguishing these entities. We quantitatively assessed the density of $\mathrm{C} 3$ deposits to objectively characterize the spectrum of deposits in $\mathrm{C} 3$ glomerulopathy.

Design: We identified all renal biopsies at Vanderbilt University for 2011-2013 with diagnosis of DDD or at least $2+$ greater intensity $\mathrm{C} 3$ deposits than other complement or immunoglobulin by immunofluorescence (IF). Only biopsies with distinct glomerular deposits by IF with corresponding deposits on EM were included. Assessment of density (black content) in deposits, red blood cells (RBC) and glomerular basement membranes (GBM) was performed using Adobe Photoshop from 8-bit grayscale digital photographs or scanned prints. Within the same photograph, deposits, uninvolved GBM and $\mathrm{RBC}$ were included to ensure equal photographic exposure. The most frequent type, densest and most diagnosis-defining deposits were assessed. Density was determined by normalizing the black content on a scale of $0-100$ based on the black content of uninvolved GBM (0) and RBC (100). Thus, allowing comparison of density scores in different images and cases. In addition, 9 usual type immune complex glomerulonephritis (UTICGN) cases were assessed.

Results: 30 cases of C3GP were identified, and 2 lacked EM images or grid availability and thus were excluded. DDD $(n=5)$ had the highest normalized density score, mean 93 (range 84-100). Two cases with a diagnosis that included features of DDD had a density score mean 76 (71 and 81$)$. $\mathrm{C} 3$ dominant deposit cases $(\mathrm{n}=14)$ had density score mean 54 (range 25-100). Proliferative GN or post-infectious GN with dominant $\mathrm{C} 3(\mathrm{n}=4)$ had deposit density score mean 64 (range 34-88). Other immune complex GN cases with dominant $\mathrm{C} 3(\mathrm{n}=3)$ had deposit density score mean 43 (range 35-53). UTICGN cases overall had lower density.

Conclusions: The measurement of deposit density decreases as expected from DDD to UTICGN. C3GN cases showed similar density to UTICGN. Cases with features suggestive of, but not diagnostic of DDD, were objectively confirmed to show intermediate density of deposits. Thus, the measurement of characteristic EM deposit density may be a helpful guideline for characterizing disease with $\mathrm{C} 3$ dominant deposits.

1689 Idiopathic Membranous Nephropathy and PLA2R Staining in African-Americans

NC Messias, PD Walker, CP Larsen. Nephropath, Little Rock, AR.

Background: Membranous nephropathy $(\mathrm{MN})$ is a relatively common glomerulonephritis most often seen in Caucasians and not well studied in the African-American (AA) population. We compared clinicopathologic features of AA patients with idiopathic MN to Caucasian patients with idiopathic $\mathrm{MN}$, including anti-phospholipase A2 receptor (PLA2R) staining. To our knowledge, this is the first study to assess PLA2R staining in the African American population.

Design: Our database was reviewed from 2009 to 2012 and 181 cases of MN in AA were identified. 58 patients had clinical information that met criteria for inclusion as idiopathic (negative for autoimmune disease, HBV, HCV, sarcoidosis, neoplasia et al). 39 previously reported Caucasian patients with idiopathic MN were used as control (CG). Clinical and pathological features were obtained from the patients' reports. Results: $\mathrm{MN}$ in AA presented at a younger age (51.9 $15.6 \mathrm{yrs})$ than in the Caucasians $(59.1+14.1, \mathrm{p}<0.05)$. Gender distribution was almost equal in $\mathrm{AA}$ (males $=53.4 \%$ ) whereas males accounted for $69.2 \%$ in Caucasians. AA had a mean creatinine of $2.4 \pm 3.8$ and mean proteinuria of $7.8 \pm 5.1 \mathrm{~g} /$ day at presentation. The CG was similar with 
a mean creatinine of $2.6+4.9$ and a mean proteinuria of $7.4+5.7 \mathrm{~g} /$ day. In AA patients, $82.2 \%(37 / 45)$ had nephrotic range proteinuria $(\geq 3.5 \mathrm{~g})$, compared to $83.3 \%(30 / 36)$ in the CG. There was no significant difference between the two groups regarding mean global glomerulosclerosis (AA 13\%, CG 14\%), and interstitial fibrosis- minimal to mild in most of the cases (AA $67 \%, \mathrm{CG} 66 \%$ ). IgG was present in all cases and $\mathrm{C} 3$ in most (AA 87.9\%, CG 92.3\%). Other antisera tested showed IgA (AA 27.6\%, CG 17.9\%), IgM (AA $22.4 \%, 17.9 \%$ CG) and C1q (AA 3.4\%, CG 5.1\%). Anti-PLA2r was positive in $37 / 58(63.8 \%)$ AA patients and though somewhat less, this was not significantly different than seen in CG $(71.8 \%)$. By electron microscopy, mesangial deposits were more frequent in AA $(40.4 \%)$ than in CG $(28.9 \%)$. Subendothelial deposits were seen in one case in AA and absent in CG. MN staging was also similar: stage I (AA 8.2\%, CG 10.3\%); stage II (AA 44.9\%, CG 53.8\%); stage III (AA 40.8\%, CG 33.3\%); and stage IV (AA 6.1\%, CG 2.6\%)

Conclusions: To our knowledge, this is the first case series detailing idiopathic $\mathrm{MN}$ and PLA2R staining in AA. PLA2R staining is present in the majority of cases of idiopathic $\mathrm{MN}$ in AA, though less frequently than in Caucasians. Idiopathic MN in AA presents at a younger age, which may have implications on long-term prognosis. Other clinical and pathological characteristics are similar to those found in Caucasians.

1690 Multinucleated Giant Cells Are a Common Marker for Anti-GBM Disease but Not ANCA-Related Glomerulonephritis

NC Messias, JM Ambruzs, PD Walker, SM Bonsib. Nephropath, Little Rock, AR.

Background: Multinucleated giant cell formation is uncommon in crescentic glomerulonephritis and in our experience, is usually predictive of anti-glomerular basement membrane antibody disease (anti-GBM). In order to validate this impression, we examined the prevalence of giant cell formation in anti-GBM disease in comparison to ANCA-related crescentic glomerulonephritis (ANCA-GN).

Design: Our database was searched for anti-GBM cases diagnosed from 2005 through 2012. Cases of ANCA-GN within the same period were used as controls. Seventy-five cases of anti-GBM and 80 cases of ANCA GN were blindly reviewed. Multinucleated giant cells (MGC) were defined as cells having 5 or more nuclei. Multiple demographic and histologic features were reviewed, including age, gender, presence of glomerular MGC, global sclerosis, number and type of crescents, crescents/glomeruli rate (\%), interstitial MGC, and interstitial fibrosis.

Results: The anti-GBM group was younger than the ANCA-GN group $(52.2 \pm 2.3$ yrs. vs. $59.9 \pm 2.1$ yrs.) and had similar gender distribution. Forty of 75 cases $(53.3 \%)$ of anti-GBM contained multinucleated giant cells, compared to 5 of $80(6.2 \%)$ ANCAGN $(p<.0001)$. MGC were seen most commonly in cellular crescents but were found in fibrocellular and fibrous crescents in both groups. The number of multinucleated giant cells per glomerulus ranged from 1-4. The presence of giant cells correlated with the percentage of crescents in the anti-GBM group, but not in the ANCA-GN group. Interstitial MGC were infrequent, but more often observed in the anti-GBM than ANCAGN group ( $14.6 \%$ vs. $2.5 \%, \mathrm{p}<.05)$. Interstitial fibrosis was mild to moderate in most cases, and did not differ between the groups (62.6\% vs.71.2\%).

Conclusions: Giant cell formation is a common finding in anti-GBM disease. MGC can serve as a marker of anti-GBM disease since they are only rarely observed in ANCAGN. The presence of giant cells correlate with a higher proportion of crescents in antiGBM disease and this is not observed in ANCA-GN. In the absence of serologic and immunofluorescence data, the presence of giant cells in a crescentic glomerulonephritis strongly suggests anti-GBM disease.

1691 Paraffin Immunofluorescence in the Renal Pathology Laboratory: More Than a Salvage Technique

NC Messias, PD Walker, CP Larsen. Nephropath, Little Rock, AR.

Background: Immunofluorescence studies on paraffin embedded tissue after pronase digestion (pIF) is usually advocated as a salvage technique in renal pathology though it has also been used to reveal masked deposits in light chain proximal tubulopathy when staining is negative on routine IF (rIF). We have found that pIF can "unmask" immunoglobulin (Ig) deposits in glomerulonephritis as well and now use pIF routinely for this purpose. The aim of this study was to evaluate the utility of pIF to avoid misdiagnosis of cases with masked Ig deposits.

Design: We reviewed our files from January through July of 2013 for all native renal biopsies in which pIF had been used. The indication for the use of pIF, and the contribution of the pIF results to the final diagnosis were assessed. pIF results were classified as follows: (A) pIF did not change the diagnosis (DX), (B) pIF made a significant contribution to the DX, confirming the presumptive light microscopy DX, e.g. negative $\mathrm{pIF}$ in ANCA cases, IgG deposits in membranous nephropathy, etc. or (C) pIF was necessary to the DX, e.g. IgA deposits in IgA nephropathy.

Results: pIF was used in $220(6.1 \%)$ of native biopsies. In 42 cases $(19.1 \%)$, pIF was used to evaluate for masked Ig deposits. These cases had features suspicious for immune complex-mediated disease on light microscopy, with no or weak immunoglobulin staining on rIF. In these cases, pIF was necessary for DX in 14 cases $(33 \%)$ and made a significant contribution in 2 cases $(5 \%)$. Prior to $\mathrm{pIF}, 8$ of the 14 cases with masked deposits had C3 only/dominant staining and could have been misdiagnosed as C3 glomerulopathy. pIF was used as a salvage technique in 156 cases $(70.9 \%)$. pIF was negative, importantly ruling out immunoglobulin, complement and light chain deposits, in 87 cases $(56 \%)$. In the remaining salvage cases, pIF was necessary for DX in 19 cases $(12 \%)$ and made a significant contribution in 48 cases $(31 \%)$. The least common indication for $\mathrm{pIF}$ was to evaluate paraprotein-associated disease ( 22 cases, $10 \%$ ). pIF was necessary for DX in one case of light chain proximal tubulopathy and important for DX in one case of light chain cast nephropathy.
Conclusions: pIF is a valuable tool for the renal pathology laboratory. In about $1 / 5$ of our cases, it was used to demonstrate potentially masked deposits, and it had impact on the diagnosis in almost half of these. We also confirmed its value as a salvage technique. Taken together, pIF was necessary or significant for diagnosis in greater than $1 / 3$ of the cases where performed.

1692 Polyoma (BK) Virus-Associated Nephropathy (PVAN) and Serum Viral Load: A Histopathological Study of Viral Replication in the Kidney Biopsy and Its Correlation with Viral Load

AV Mikhailov, F Salem. Mount Sinai Medical Center, New York City, NY.

Background: Polyomavirus- associated nephropathy (PVAN) is a significant cause of premature allograft failure. Early diagnosis of PVAN is vital to ensure prevention of irreversible renal damage; however diagnosis relies primarily on allograft biopsy. Our aim is to determine if the viral load measurement can be used to predict the severity of PVAN.

Design: 53 kidney allograft biopsies diagnosed with PVAN, between the period 2009 to 2013 were evaluated. All patients had BK viral load determined by plasma PCR method within 2 weeks of kidney biopsy. Each biopsy was variably positive for SV40 imunoperoxidase stain. The histological pattern of PVAN was determined as previously described by Drachenberg et al. The BK virus replication in the kidney was detected by SV40 immunostain and quantified as pattern S1 (less than $25 \%$ of renal parenchyma affected), S2 (26 to $50 \%$ of parenchyma affected), and S3 (more than $50 \%$ of parenchyma affected). The significance of differences between patient biopsy groups was determined using single factor ANOVA (Figure 1; error bars, standard error of the mean).

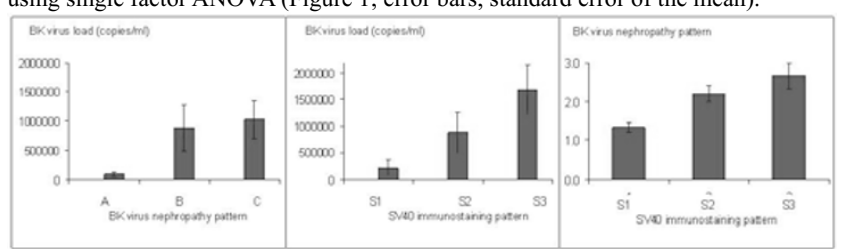

Results: The histology of PVAN in the biopsies corresponded to Drachenberg patterns A (viral cytopathic changes) (20 patients), B (28 patients) (tubular atrophy/inflammation/ fibrosis) and $\mathrm{C}$ (diffusely scarred renal tissue) (5 patients). The pattern of renal damage tended to correlate with the viral load (Figure 1, left); the difference between the BK nephropathy pattern groups had borderline statistical significance $(p=0.06)$. The involvement of the renal cortex with the BK virus replication was directly associated with the amount of the virus in the blood (Figure 1, middle) $(\mathrm{p}<0.05)$. Biopsies showing larger areas of BK virus replication in the cortex (S2 and S3) presented with more severe patterns of BK nephropathy (Figure 1 right, $\mathrm{P}<0.06$

Conclusions: The BK viral load can be used to predict the amount of viral replication in the kidneys and the extent of renal damage in transplant patients.

1693 CD28-/CD8+ Subset of Lymphocytes May Escape Co-Stimulation Blockage with Belatacept to Mediate Acute Cellular Rejection in Allograft Kidneys

D Ng, S Geiger, G Szabo, K-Y Jen, FG Vincenti, ZG Laszik. UCSF Medical Center, San Francisco, CA; KIT, Karlsruhe, BW, Germany.

Background: Belatacept (Bela), a CD28/B7 co-stimulation pathway inhibitor, used as an alternative to calcineurin inhibitor (CNI)-based immunosuppression after renal transplantation, is associated with a higher incidence of acute cellular rejection (ACR). Recent experimental data indicate that such ACR might be mediated by alloreactive CD8+ memory effector T cells featuring low CD28 expression. Our study tested the hypothesis that in kidney biopsies with ACR the proportion of infiltrating CD8+ lymphocytes co-expressing CD28 is lower in patients treated with Bela than those treated with CNI.

Design: Frozen kidney biopsies with ACR from patients on Bela $(n=14)$ or CNI $(n=11)$ were co-stained with CD8 and CD28 double immunofluorescence (IF). To assess the co-expression between the two markers, M1 and M2 Manders' Coefficients, representing the fraction of CD8 positivity overlapping CD28 positivity and the fraction of CD28 positivity overlapping CD8 positivity, respectively, were calculated from multiple representative 40x digital images taken from the renal cortex. The M1 and M2 Manders' Coefficients were compared between the Bela and CNI groups using Student's T-test. Results: Due to differences in the IF staining pattern with CD8 (continuous membrane) and CD28 (punctate) there was an imperfect overlap between $\mathrm{CD} 8 / \mathrm{CD} 28$ double positive cells resulting in non-correctable relative underestimation of the CD28+ population. Clustering of the CD28 signal was apparent and prominent differences in the CD28 signal intensity within the same biopsy were suggestive of a heterogeneous CD28 and possibly heterogeneous CD8 population. The M2 Coefficient was significantly higher $(\mathrm{p}=0.01)$ in the CNI group (0.55) than in the Bela group (0.3), indicating a higher proportion of CD8+ cells that are CD28- in the Bela group. There were substantial, although statistically insignificant $(\mathrm{p}=0.09)$ differences in the M1 Coefficient between the two groups ( 0.22 in Bela vs. 0.08 in $\mathrm{CNI})$

Conclusions: The higher proportion of CD28-/CD8+ tissue infiltrating lymphocytes of allograft kidneys with ACR in patients treated with Bela is suggestive of potential differences in the pathogenetic mechanisms of ACR from those seen in patients on CNI. Consistent with experimental data these findings may indicate that the Bela-associated ACR is mediated (at least in part) by CD28-/CD8+ memory effector T cells escaping co-stimulation blockage with Bela. Further studies to address the relationship of CD28 expression with additional $\mathrm{T}$ cell subpopulations are warranted. 
1694 Classification of Polyomavirus Nephropathy: A Proposal from a Multicenter Study

V Nickeleit, S Seshan, AM Gasim, H Singh. The University of North Carolina, Chapel Hill, NC; Cornell University, New York Presbyterian Hospital, New York, NY.

Background: Polyomavirus nephropathy $(\underline{P V N})$ is the most important renal allograft infection with an incidence of $4 \%$ in 2010. It can cause pronounced chronic graft failure in $>50 \%$ of patients reported in some series. Since PVN is not routinely subclassified into different disease stages, it is unclear whether specific morphologic PVN phenotypes carry prognostic significance. The AIM of this multicenter study is to define clinically predictive morphologic PVN disease stages in a large cohort of diverse patient populations.

Design: Nine US and European centers collected 192 diagnostic index biopsies with PVN from 192 patients; 90\% transplanted between 2003-2008 (74\% males, 31\% blacks). Histologic changes were evaluated (including central pathology review) at time of initial diagnostic index biopsy (Banff scores, intra renal PV load levels (pvl) based on percentage of tubular SV40-T staining). Clinical data were collected from time of transplantation until 24 months post index biopsy. Statistical analyses included hierarchical mixed models, stepwise regression and placed specific emphasis on racial backgrounds.

Results: Focusing on renal function and graft survival as clinical criteria of interest strongest associations were found with intra renal pvl and Banff ci interstitial fibrosis scores (grouped into minor: $<25 \%$ fibrosis, and major: $>25 \%$ ). Limited associations were seen with the presence or absence of intra nuclear PV inclusion bodies. Based on these statistics, 3 PVN disease grades were defined: grade 1 (minimal - pvl $<1 \%$ and ci $<25 \%$ ), grade 2 (florid - pvl and ci scores other than listed in grades 1 and 3 ), and grade 3 (advanced - pvl $>10 \%$ and $\mathrm{ci}>25 \%$ ). $24 \%$ of cases $(46 / 192)$ were PVN grade $1,64 \%$ grade 2 , and $13 \%$ grade 3 . PVN grades correlated significantly with: a) time of diagnosis (earliest at a median of 18 months in grade 1), b) peak serum creatinine level at time of index biopsy (worst with $2.6 \mathrm{mg} / \mathrm{dl}$ in grade 3 ), 3) allograft function post index biopsy (best in grade 1 with stable function and a minimal delta change compared to baseline readings), 4) PVN disease healing (best with $79 \%$ in grade 1 ), and 5) allograft failure (worst in grade 3 with $50 \%$ ); all $\mathrm{p}<0.001$.

Conclusions: The subclassification of PVN into 3 disease grades (minimal, florid, advanced) helps to predict graft function, graft survival and the resolution of PVN. PVN classification can help with managing kidney transplant recipients and improving comparative analyses of clinical trials.

1695 High Rate of Detection of Medical Renal Disease in Selected Tumor Nephrectomies Referred to Renal Pathology Division

K Park, S Salvatore, B Robinson, M Shevchuk, JM Mosquera, S Seshan. Weill Cornell Medical College, NY, NY

Background: Recent retrospective studies indicate evaluating non-tumor portions of tumor nephrectomy specimens is useful in diagnosing non-neoplastic renal disease which has prognostic significance. Following the 2010 CAP guidelines, the evaluation and implications of these non-neoplastic findings at the time of surgery may be useful in clinical management post-operatively in patients with reduced renal mass.

Design: From January 2011- August 2013, there were 335 nephrectomies performed at our institution, 190 partial and 145 total. All were evaluated using a PAS special stain by the Genitourinary Pathology Division, and $84(25 \%)$ were referred to the renal pathology division based on clinical parameters (hypertension, diabetes, chronic renal insufficiency), young age of patients ( $<40 \mathrm{yrs}$ ), and findings based on light microscopic pathologic changes (especially when discrepant from the age of the patient). Of these, proteinuria was known in 11 cases and hematuria in 20 (persistent pre-op hematuria in 2). Electron microscopy was performed in 8 cases. Global glomerulosclerosis, tubular atrophy/interstitial fibrosis, and vascular sclerosis (scored from 0-3) were evaluated in each case. Follow-up creatinine levels were recorded for cases with severe vascular sclerosis, diabetic nephropathy, or immune complex disease.

Results: Of the 84 cases, 7 cases had end-stage renal disease (ESRD) at the time of resection, 3 due to diabetic nephropathy (DN), 1 lupus, and 3 due to unclear etiology. Of the remaining 77, 11 had DN, 3 immune complex glomerulonephritis (ICGN), 2 active interstitial nephritis (AIN), 1 atheroemboli, 1 obesity-related glomerulopathy, 2 thin glomerular basement membranes, 1 thrombotic microangiopathy, and 10 obstructive nephropathy. 2 also showed medullary urate nephropathy. 22 had only severe vascular sclerosis. In all, 68\% (53/77) of cases sent for consultation had significant pathologic findings, while $32 \%$ (24) had minimal pathologic changes. Six patients were referred to a nephrologist after nephrectomy. Two patients developed ESRD following surgery, from DN and vascular sclerosis, after 1-2 years.

Conclusions: Close interaction between urological and renal pathology with careful evaluation and selection of non-neoplastic portion of tumor nephrectomies for second opinion has a high yield in detection of medical renal disease. Real time assessment of cases is practical and useful for further clinical management of patients following surgery with prompt referral to the nephrologist by the urologist.

1696 Proliferative Glomerulonephritis with Monoclonal IgG Deposits and Associated Hematopoietic Neoplasms

P Paueksakon, AB Fogo. Vanderbilt University, Nashville, TN.

Background: Proliferative GN with monoclonal IgG deposits (PGNMID) is a rare form of renal involvement by monoclonal gammopathy that mimics immune complexglomerulonephritis. We reported on our experience of PGNMID and association with a broader spectrum of hematopoietic abnormalities than previous reported.

Design: All patients with proliferative GN (excludes patients with SLE) in our 19952013 renal biopsy files at VUMC were reviewed. Of these, $\kappa$ and $\lambda$ immunofluorescence studies were not performed in 233 patients and 64 of theses had available frozen tissue for additional $\kappa, \lambda$, and IgG subclass immunofluorescence studies. Clinical and morphological data of theses patients with monoclonal IgG deposits were then reviewed. Results: A total of 1,110 patients had proliferative GN, and of these, 12 patients $(1 \%)$ were identified as PGNMID. The mean age at diagnosis was 53 years. The patients were mostly white (75\%), and male: female ratio was $1.4: 1$. Clinical presentation included proteinuria and hematuria in all, nephrotic syndrome (58\%), and renal insufficiency $(50 \%)$. There were 9 patients with available serum monoclonal protein data. We identified monoclonal serum protein in 2 patients $(22 \%)$ and of these, one had chronic lymphocytic leukemia and the other had no hematologic malignancy. Of the remaining 7 patients with no monoclonal serum protein, one had B-cell lymphoma. None of these 12 patients with PGNMID had myeloma. Histologic patterns were MPGN (84.0 $\%$ ), proliferative $\mathrm{GN}$ with membranous features $(8 \%)$, and $\mathrm{GN}$ with predominantly membranous features $(8.0 \%)$. The most common single light-chain isotype and single heavy-chain subtype was IgG3k (67\%) followed by, IgG3 $\lambda(17 \%)$, IgG1k $(8 \%)$, and IgG1 $\lambda(8 \%)$. Follow up for 7 patients with available data showed that, none had complete remission, $1(14 \%)$ had partial remission of proteinuria, $2(29 \%)$ had persistent renal dysfunction, and $4(57 \%)$ progressed to ESRD and received kidney transplant and all developed recurrent disease at 2-10 months later. No monoclonal protein was detected in these 4 patients with recurrent PGNMID. No clinical or laboratory evidence of cryoglobulinemia was found in our series.

Conclusions: PGNMID is very rare. Monoclonal protein was found in our series in $22 \%$ similar to the previous reports and hematologic malignancy is rare. No myeloma was found in our series. We add B-cell lymphoma to the spectrum of underlying disease in PGNMID. Further, our data suggest very high risk for recurrence of PGNMID in the transplant despite the absence of serum monoclonal protein.

\section{Renal Recovery from Delayed Graft Function Is Not Predicted by} Ki-67, IL-18, and Lectin Staining

SP Salvatore, DK Kannabhiran, C Hartono, SV Seshan. Weill Cornell Medical College, New York, NY.

Background: There are diverse etiologies of acute renal injury in the immediate post-renal transplant period including ischemic acute tubular injury (ATI), rejection, medication toxicity, and donor-related disease or early recurrent disease. Recovery may depend on the type, extent and severity of the injury. Biopsy results may help guide treatment and predict future graft function.

Design: Transplant kidney biopsies from 2000 to 2010 (1777 total) were retrospectively reviewed from patients biopsied within the first month following transplantation (69, $4 \%$ ). Immunohistochemical stains for Ki-67, IL-18, EMA, and lectins were performed in a subset to analyze the tubular epithelial injury and potential for recovery.

Results: The biopsy findings were ATI in 20 (17 mild and 3 severe), calcineurin inhibitor toxicity 18 , antibody mediated rejection (AMR) 16, acute cellular rejection 7 , mixed rejection 1 , vascular rejection 1 , active interstitial nephritis 2 , focal segmental glomerulosclerosis 2, diabetic nephropathy (DN) 1, and pyelonephritis 1 . A subset of 28 cases, from donors 22-75 years old, representing all diagnoses were analyzed. Donors were 1-67 years old. Four were pediatric and 6 were extended criteria donors (ECD), over 60 years. 21 were deceased donors (mean cold ischemia time 23hrs (range: 10$38)$ ) and 7 were living donors. 18/28 required immediate hemodialysis, ranging from 1-109 days duration. 9/28 transplants failed (with mean 3.6 years follow-up), 6 resulted in primary permanent non-function (3 ATI [ 1 mild, 2 severe], 2 AMR, and 1 mixed rejection), mean Ki67 11.5 $\pm 6.4 \%$, and 3 subsequent failure up to 4 years later (1 AMR, 1 recurrent DN, 1 unknown), mean Ki67 17.5 $\pm 19.8 \%$. The remainder had creatinine 0.8-3.5 mg/dL (mean 1.7) with 4 months to 7 years follow-up, mean Ki67 8.5 \pm 8.6 . 3 patients had low mean arterial pressure $(<80)$ prior to transplant, of whom all had primary allograft non-function. 2/4 ECD kidneys failed (1-immediate, 1-4 years later). The case with the longest dialysis requirement had $0 \%$ Ki67. IL-18 and lectin staining did not reveal a significant difference between diagnoses, outcomes, or other variables. Conclusions: Allograft dysfunction in the immediate post-transplant period can have variable etiologies and outcomes, poorest for AMR and severe ATI. The largest subset, mild ATI, is presumably related to ischemic tubular injury and has heterogeneous epithelial regenerative change and subsequent recovery. Ki-67 may help predict time to response.

\section{The Renal Pathological Findings in Diabetic Patients with Acute}

\section{Renal Failure}

SP Salvatore, SV Seshan. Weill Cornell Medical College, New York, NY

Background: Diabetic nephropathy (DN) is the most common cause of end stage renal disease in the U.S. Typically, presenting with proteinuria and progressive renal insufficiency, the diabetic patient may be managed clinically without a diagnostic kidney biopsy. However, acute renal failure in the diabetic patient tends to have many etiologies requiring a kidney biopsy for definitive diagnosis

Design: Of 7157 native kidney biopsies from 2000-2013, 837 (11.7\%) had diabetic nephropathy and 458 of those $(54.8 \%)$ presented with acute renal failure (ARF) with or without significant proteinuria and hematuria. ARF was defined by KDIGO criteria ( $>0.3 \mathrm{mg} / \mathrm{dL}$ increase in serum creatinine within $48 \mathrm{hrs}$ or $50 \%$ increase within 7 days). Analysis of the clinicopathologic features of this cohort was performed.

Results: Renal biopsy findings of DN are increasing in recent years in our cohort, present in $8.4 \%$ of cases from $2000-2004$ and $16.4 \%$ from $2011-2013$. The 458 patients with ARF ranged from $14-88$ years old and were $58 \%$ male. The average presenting creatinine was $4.6 \mathrm{mg} / \mathrm{dL}(1.5-22)$, with 8 patients on dialysis, and proteinuria was $6.6 \mathrm{~g} / \mathrm{d}(0-34)$. A second diagnosis in addition to diabetic nephropathy was rendered in 309 cases $(67 \%)$, most commonly patchy or diffuse active interstitial nephritis (AIN) (26.4\%), post-infectious glomerulonephritis (PIGN) (10.5\%), acute tubular injury $(10.9 \%)$, podocytopathy $(5.7 \%)$ [23 collapsing glomerulopathy, 10 minimal change, 6 FSGS], crescentic GN (2.6\%), thrombotic microangiopathy $(2.2 \%)$, and occasional 
membranous GN (5 cases), IgA nephropathy (3), Bence Jones cast nephropathy (2), lupus nephritis (4), atheroembolic disease (3), amyloidosis (2), cryoglobulinemia (1), and membranoproliferative GN (3). The cases without a superimposed disease process (33\%) showed advanced diabetic disease, class III (nodular diabetic glomerulosclerosis) $39 \%$ to class IV (having $>50 \%$ global GS) $36 \%$, with interstitial fibrosis $(65 \%$ ), and severe vascular disease $(42 \%)$

Conclusions: DN is increasingly found in the renal biopsy population. Acute renal failure, a common but important indication for kidney biopsy in a diabetic patient, is frequently associated with a superimposed disease but may also disclose advanced diabetic nephropathy alone. In these patients, renal biopsy yields diagnostic and prognostic information for appropriate management

1699 Renal Pathology in Immunodysregulation, Polyendocrinopathy, Enteropathy, X-Linked (IPEX) Syndrome

$Y$ Sheykin, NRodig, B Schmidt, P Lee, S Rosen. Beth Israel Deaconess Medical Center, Boston, MA; Children's Hospital of Boston, Harvard Medical School, Boston, MA.

Background: Autoimmune polyglandular syndromes are a group diseases of which IPEX is the most lethal. IPEX is caused by mutations in the gene on chromosome $\mathrm{X}$, encoding the fork-head box P3 (FOXP3) transcription factor that governs the normal functioning of regulatory $\mathrm{T}$ cells. The syndrome has been characterized by multiple endocrinopathies, enteropathy and dermatitis. Different forms of renal injury have also been occasionally reported including "tubulonephropathy", interstitial nephritis, minimal change and membranous glomerulonephritis (GN), but these descriptions have been very limited.

Design: Here we report three patients with IPEX and renal manifestations from the archives of Children's Hospital of Boston.

Results: The index patient is a 1-month-old male who presented with renal failure, high grade proteinuria, vesiculopustular rash and diarrhea. Renal biopsy showed endocapillary proliferative GN with deposition of immune complexes in glomeruli (membranous pattern), tubular basement membranes and vessels, as well as interstitial nephritis. Skin biopsy showed interface dermatitis, IgG deposition at the dermoepidermal junction, sweat gland basement membranes and vessels, as well as circulating antibodies to collagen type VII, features that have been described in bullous SLE. Flow cytometric analysis showed virtually absent FOXP3 expression on CD4+CD25+ T cells. The second patient was a male with associated Evan's syndrome, diabetes and severe eczema. Initial renal biopsy at the age of 1 showed membranous GN with extensive reparative changes. Renal biopsy at the age of 3 showed endocapillary proliferative GN with immunofluorescence and electron microscopy findings of a membranous type with an IgG tubular basement membrane granular pattern. A missense mutation of FOXP3 was confirmed by genetic analysis. The remaining patient is a 10-year-old male with associated seronegative autoimmune hepatitis, who at the age of 7 months presented with nephrotic syndrome due to a typical membranous GN and IgG granular tubular basement membrane staining. He had virtually absent FOXP3 + T cells and a mutation in exon 7 of the FOXP3 gene. At the age of 7 he underwent a stem cell transplant followed by resolution of his renal symptoms.

Conclusions: Taken together, glomerular and tubular basement membrane immune complex deposition in all 3 patients presents a distinctive combination, which under usual circumstances would be consistent with SLE, the classic disease of T-cell dysfunction and autoimmunity.

1700 Polyomavirus Nephropathy from Diagnosis to Disease Clearance and the Impact on BANFF Activity and Chronicity Indices over Time

HK Singh, VNickeleit. The University of North Carolina at Chapel Hill, Chapel Hill, NC. Background: The morphologic changes induced by persistent Polyomavirus Nephropathy (PVN) have not been systematically studied. It is unknown how significantly PVN contributes to chronic allograft injury over time especially in patients with disease clearance who do not progress to endstage graft failure.

Design: We evaluated paired renal biopsies for cause following standard protocols from 14 patients ( $\mathrm{n}=28$ total biopsies) at the time of initial $\mathrm{PVN}$ diagnosis and subsequently at time of PVN clearance. Mean time in weeks between biopsies was 128 (range=3.4-392 weeks). All biopsies were $\mathrm{C} 4 \mathrm{~d}$ negative by Immunofluorescence staining. At time of index biopsy: 9 cases were PVN grade 1 (minimal virally induced injury) and 5 were PVN grade 2 (florid virally induced injury). Standard BANFF criteria were used to score all biopsies. Serum creatinine levels ( $\mathrm{SCr}$ ) were used to assess renal function. Clearance of PVN was defined as no immunostaining for the SV40 large T antigen and a negative urine PV-Haufen test. Statistical analysis was performed using the Wilcoxon rank-sum test.

Results: BANFF activity scores (i, ti, t, ptc, g, v) showed no statistically significant differences between index and clearance biopsies (Figure 1A). BANFF chronicity scores demonstrated increased chronic changes in clearance biopsies associated with rejection (cg), hypertension and/or calcineurin inhibitor induced toxicity (ah, cv) (Figure 1B). Renal Function-index biopsy: $\mathrm{SCr}(\mathrm{mg} / \mathrm{dL})$ mean=3.22, range 1.5-7.22; Renal Functionclearance biopsy: $\mathrm{SCr}(\mathrm{mg} / \mathrm{dL})$ mean=2.29, range 1.1-3.9.

\section{Mean Acute Scoring: PVN Index and Clearance Biopsies}

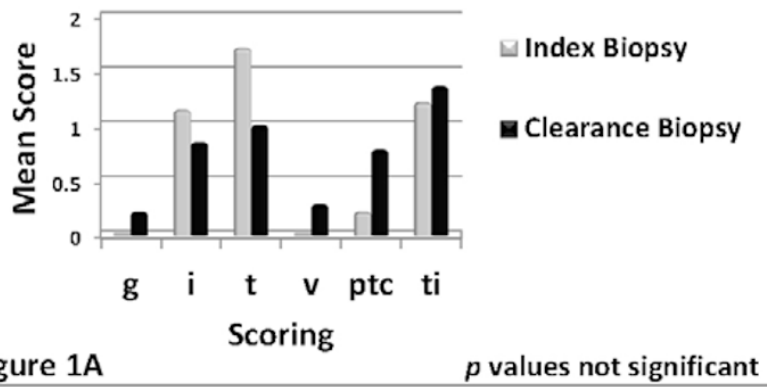

\section{Mean Chronic Scoring: PVN Index and Clearance Biopsies}

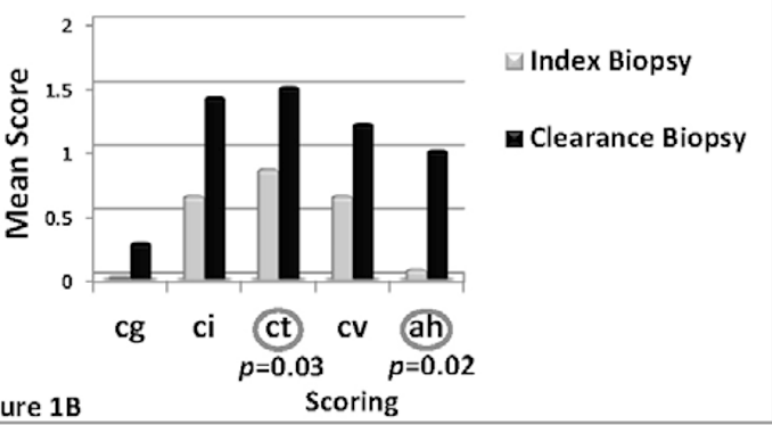

Conclusions: In cleared PVN grades 1 and 2, there was improvement in renal function compared to time of PVN index biopsy. Patients who cleared PVN showed an increase in chronic changes that were at least in part caused by chronic rejection and hypertension induced arterionephrosclerosis. If PVN grades 1 and 2 heal, then viral nephropathy does not seem to significantly and independently contribute to chronic allograft injury.

1701 Diffuse Diabetic Glomerulosclerosis (DGS) Is Not and Never Has Been Diffuse or Sclerotic

LC Stout. University of Texas Medical Branch, Galveston, TX.

Background: Diabetic nephropathy has 2 forms, DGS and nodular glomerulosclerosis. The landmark 1959 study (DD Gellman, CL Pirani, JF Soothill et al. Diabetic Nephropathy: A Clinical and Pathologic Study Based on Renal Biopsies, Medicine, 38:321-367, 1959) established DGS as the most important, being far more common and more highly associated with renal insufficiency. Their initial DGS lesion was a segmental ring like thickening of peripheral capillary walls by "deposits" of pink material that incorporated endothelial cells and podocytes and obscured the glomerular capillary basement membrane (GBM) as it expanded. They could not find a late stage DGS lesion and speculated that the initial process continued until the tuft was occluded. They also found that DGS was neither diffuse nor sclerotic but retained the name, the diffuse part of which apparently convinced most that DGS had a biochemical rather than a hydraulic etiology.

Design: None.

Results: Their initial lesions were called segmental mesangial expansions (SMEs) in our study of 74 consecutively autopsied diabetic ( 25 with any diabetic lesions-end stage cases excluded) and 59 matched controls. A 3 dimensional $4 \mu$ and $1 \mu$ serial section study of 19 SMEs in 7 of 10 cases with minimal to mild DGS found that the lack of GBMs in their initial lesions was due to mesangial interposition (MI) extending from the feeding capillary into tortuous branches with tiny lumens and no GBMs that formed the solid appearing SME body before joining capillaries at the SME periphery, some of whose GBMs came from the feeding capillary. MI was found to be normal mesangium by ultrastructural step sections in 1 case. MI also probably caused segmental collapsing capillary tufts (SCCT) in 2 of 25 diabetics by extending into and gradually occluding 1 leg of a capillary bifurcation. Some SCCT up to $35 x 60 x>55 \mu$ were completely autolyzed and thought to fall off the tuft causing lobulation. The type 1 diabetic had 16 SCCT seen in $1 \mu$ but not $4 \mu$ sections, so SCCT may be under recognized. Above data are unpublished observations.

Conclusions: MI may be a normal response to increased flow/and pressure? from hyperperfusion since structural connections between capillary walls and mesangium, and small efferent arteriolar muscles indicate that capillary flow is mainly controlled by mesangium (M Elger,T Sakai, W Kriz. The Vascular Pole of the Renal Glomerulus of Rat. Advances in Anatomy, Embryology and Cell Biology, vol 139. Berlin, Germany: Springer-Verlag; 1998. p. 1-97. MI might cause additional damage by reducing free wall filtering surface and increasing mesangial centripetal flow. 
1702 Identification of Biomarkers of Diabetic Nephropathy Progression in Renal Transplant Patients Using Novel Chemical Imaging Approaches VK Varma, A Susma, A Kajdacsy-Balla, S Akkina, S Setty, $R$ Bhargava, MJ Walsh. University of Illinois at Chicago, Chicago, IL; University of Illinois at UrbanaChampaign, Urbana, IL.

Background: Kidney transplantation is the main treatment for end-stage renal disease, however close monitoring of post-transplant biopsies is required to identify subclinical complications. In high risk patients surveillance biopsies are acquired every 6 to 12 months post transplantation to examine tissue histology for complications. In this study, we focused on identifying biochemical markers associated with recurrent diabetic nepthropathy using Fourier Transform Infrared (FT-IR) spectroscopic imaging. FTIR imaging is an emerging approach to obtain label-free images of the biochemical composition of tissue biopsies (including proteins, lipids, collagen, DNA and glycation). Design: Initial studies identified 8 patients with no diabetic nephropathy and 8 patients with diabetic nephropathy. Serial sections were stained with PAS or imaged using FTIR. IR spectra were extracted from the different glomerular and tubular structures and compared to identify biomarkers associated with diabetic nephropathy progression. A second study identified five transplant patients with repeat biopsies who underwent rapid recurrent diabetic nephropathy and five normal patients with no evidence of diabetic nephropathy. Biochemical information was extracted from the images to identify chemical changes associated with the progression of diabetic nephropathy. Results: A number of biomarkers were identified that were shown to change in glomerular and tubular structures associated with increasing severity of nephropathy due to diabetes. The most robust biomarkers of disease progression were increased levels of glycation and the symmetric phosphate band in glomerular structures. These two biomarkers were also consistently found to be increasing in the cohort of patients that had multiple surveillance biopsies and underwent rapid diabetic nephropathy recurrence suggesting that these features may be predictive of early diabetic nephropathy changes. Conclusions: FT-IR imaging is an emerging approach that can allow for novel insight into the biochemical changes associated with disease progression in transplant renal biopsies. We have identified a number of biomarkers associated with the advancement of diabetic nephropathy and that we can track the early recurrence of diabetic nephropathy in surveillance biopsies. FT-IR imaging is potentially powerful adjunct to current pathological methods allowing for the label-free and rapid identification of early markers of transplant complications.

1703 Identifying Iron Deposition in Proximal Tubules Is a Useful Method to Distinguish Sickle Cell Disease (SCD) Associated Renal Tubular Injury from Other Injury Etiologies

$Y$ Wang, SC Wolforth, M Doshi, S Khan, MT Rooney, WLi, PL Zhang. William Beaumont Hospital, Royal Oak, MI; Detroit Medical Center, Detroit, MI

Background: Sickle cell nephropathy (SCN) has been thought to be a vascular disorder since blood clot formation from sickle cells is considered to be the cause of renal disease. We hypothesize that sickle cells, known vulnerable for broken, may dump large amount of iron to proximal tubules and cause renal tubular injury, thus partially contributing to renal failure seen in sickle cell nephropathy. We compared iron stains in 3 cases with SCD and 29 controls.

Design: Three biopsies from patients with SCD (one native and two transplant biopsies) were stained for iron using conventional Prussian blue method. The proximal tubules were evaluated for iron staining ( 0 to $3+)$ and correlated with clinical scenarios. Controls include 12 IgA nephropathy, 13 thrombotic microangiopathy (TMA) and 4 "pure" acute tubular necrosis (ATN) cases were also stained for iron.

Results: All controls showed negative or minimal iron staining in proximal tubules, except two TMA with $2+$ iron staining in proximal tubules. The first native biopsy with SCD showed membranoproliferative pattern of glomerulopathy and $3+$ iron staining in proximal tubules, consistent with a SCN. The second patient, status post renal transplant 2 years ago, developed acute renal failure, and his renal biopsy was found to have acute tubular injury (ATI). Diffusely increased iron staining (2+) in proximal tubules was present, thus the ATI was most likely resulted from iron toxicity to proximal tubules, and EM confirmed aggregated sickled RBC in glomeruli, indicating a recurrent $\mathrm{SCN}$. The third patient, status post renal transplant 4 years ago, developed acute renal failure and positive donor specific antibody. His renal biopsy revealed 1a acute cellular rejection, diffuse positive $\mathrm{C} 4 \mathrm{~d}$ in peritubular capillaries and thrombotic microangiopathy (TMA). The iron staining was focally and weakly present, implying that the TMA was most likely associated with the acute antibody mediated rejection (AAMR, type 2) rather than recurrent SCN.

Conclusions: Our data indicate that iron staining is a non-expensive but effective method in distinguishing SCD associated renal injury from other etiologies, supporting the view that iron overloading in proximal tubules could be a cause of ATI.

1704 Hic-5 and Glomerular Senescence in Chronic Proteinuric Glomerulopathies

A Weins, P Mundel. Brigham and Women's Hospital, Boston, MA; Massachusetts General Hospital, Boston, MA

Background: Diffuse diabetic glomerulosclerosis (DDG) and Primary Focal Segmental Glomerulosclerosis (FSGS) are patterns of glomerular injury clinically characterized by slow progression and proteinuria. To date little is known about molecular mechanisms involved in the evolution from minimal proteinuria and mild podocyte injury to nephron loss. This study explores a role of the focal adhesion protein hic-5 in this process by focusing on hic- 5 expression in glomerular mesangial and epithelial cells. The findings build on our recently published results on the pathogenesis of progressive glomerular lesions.
Design: We performed immunohistochemical analysis of archived kidney biopsies from patients with DDG and FSGS, and control cases (including Minimal Change Disease, IgA Nephropathy, Vascular Disease and Acute Tubular Injury). In addition, mouse models of chronic glomerular disease (AT1R transgenic rats, murine ADR nephropathy), mouse models of acute glomerular disease (Protamine Sulfate perfusion and injection of Nephrotoxic Serum) and conditionally immortalized podocytes were used to explore the role of hic-5 in chronic versus acute glomerular injury.

Results: While hic-5 expression is limited to vascular smooth muscle cells in control cases, glomeruli in DDG and FSGS show specific and robust up-regulation of hic-5 in mesangial and in scattered glomerular epithelial cells. In addition, the same samples also reveal features of cellular senescence in occasional glomerular epithelial but not mesangial cells, as confirmed by focal low-level expression of $\mathrm{p} 53$ and positive SA-betagalactosidase staining. Conditionally immortalized podocytes with up-regulation of hic5 at focal adhesions show increased adhesiveness to the ECM and concomitant features of cellular senescence. Glomeruli of AT1R tg rats and of mice with ADR nephropathy, both models of progressive glomerular injury, also reveal substantial up-regulation of hic-5 compared to their wild type littermates. In contrast, hic-5-/- mice show neither protection nor worsening of acute podocyte FPE or proteinuria compared to wild type littermates after perfusion with protamine sulfate or injection of Nephrotoxic Serum. Conclusions: These findings suggest a link between hic- 5 and glomerular senescence in progressive glomerular injury. Understanding if adhesion-dependent induction of senescence may promote intraglomerular cross talk, and how it participates in accelerating or decelerating disease progression is the focus of further studies, and may help to identify novel and specific therapeutic targets.

1705

Mice

$J$ Yang, $H$-C Yang, AB Fogo. Vanderbilt University Medical Center, Nashville, TN. Background: Plasminogen activator inhibitor-1 (PAI-1) prevents matrix degradation by inhibiting plasmin formation and regulates cell function by the vitronectin pathway. We previously showed that systemic PAI-1 knockout mice were resistant to glomerulosclerosis after 5/6 nephrectomy. In vitro, PAI-1 deficient podocytes had reduced apoptosis and preserved cytoskeleton after injury compared to wild type. In this study, we therefore investigated whether conditional podocyte PAI-1 knock-out could protect against glomerulosclerosis in a primary podocyte injury model.

Design: By mating PAI-1 $1^{\text {loxp}} /$ Teto-Podocin $\mathrm{Cr}^{+}$mouse with PAI- $1^{\text {loxp }} / \mathrm{Nep} 25$ mouse, we generated conditional PAI-1 knock-out mice (KO; PAI-1 ${ }^{\text {loxp/loxp }} / \mathrm{Teto}^{-P}$-Podocin $\mathrm{Cre}^{+} /$

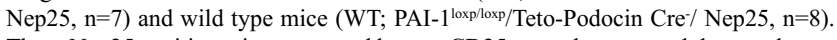
These Nep25 positive mice expressed human CD25 on podocytes, and thus, podocyte injury and a primary FSGS can be induced by injecting the LMB2 toxin which binds CD25. On Day -7 , doxycycline $(2 \mathrm{mg} / \mathrm{ml})$ was added to drinking water to induce podocyte knock-out of PAI-1. On Day 0, LMB-2 (8 ng/g BW, I.V.) was given. Mice were sacrificed and kidneys were harvested on Day 10 .

Results: Both groups showed similar edema, reflected by increased body weight. KO had a trend to increased blood pressure vs WT (change vs baseline KO $18.8 \pm 7.0 \mathrm{vs}$. WT $1.5 \pm 4.9 \%, \mathrm{p}=0.061$ ). Proteinuria was similar between groups (urine protein:creatinine ratio WT $463.2 \pm 72.1, v s . \mathrm{KO} 496.9 \pm 49.6 \mathrm{mg} / \mathrm{ug}$, pNS). Podocyte injury was numerically increased in WT (semi-quantitative score, 0-4, WT 2.78 \pm 0.11 , vs. KO 2.26 \pm 0.27 , $\mathrm{p}=0.084$ ). Synaptopodin expression, a marker of differentiated podocytes, was higher in KO $v$. WT (glomerular positive area $\%$, KO $3.82 \pm 0.87$, vs. WT $1.98 \pm 0.23 \%, \mathrm{p}<0.05$ ). The glomerulosclerosis score and \% collagen IV area in glomerulus were not different between groups (WT $12.80 \pm 1.19$, vs. KO $14.47 \pm 0.90 \%$, pNS).

Conclusions: Knock-out of PAI-1 in podocytes results in better maintenance of podocytes after injury, but does not appear to be sufficient to prevent glomerulosclerosis or matrix accumulation in vivo. Taken together with previous systemic PAI- $1^{-/}$data, these findings support that additional glomerular or infiltrating cell PAI-1 is crucial for perpetuating sclerosis after injury.

\section{Liver}

\section{Loss of ARID1A Expression Is a Late Stage Event in Tumor} Progression of Hepatocellular Carcinoma

$H$ Abe, A Hayashi, A Kunita, J Shibahara, M Fukayama. the University of Tokyo Hospital, Tokyo, Japan; Graduate School of Medicine, The University of Tokyo, Tokyo, Japan.

Background: AT-rich interactive domain 1A(ARID1A) is a subunit of Switch/Sucrose non-fermentable (SWI/SNF) chromatin remodeling complex. Frequent mutation and loss of protein expression were reported in various carcinomas such as ovarian clear cell carcinoma and Epstein-Barr virus associated gastric carcinoma. Recently, genome-wide whole exome sequencing revealed frequent mutation of ARID1A in hepatocellular carcinoma. However, the clinicopathological significance and the role in carcinogenesis were unknown.

Design: In this study, immunohistochemistry of ARID1A was performed in tissue microarray of 290 hepatocellular carcinomas, and clinicopathological significance was analyzed. In addition, distribution of ARID1A-lost carcinoma cells within the tumor was examined by immunohistochemistry of whole sections. Expression of ARID1A in 19 dysplastic nodules was also examined.

Results: Eleven (3.8\%) of 290 cases showed loss of ARID1A expression. Loss of ARID1A expression has no significant correlation with age, sex, HBV or HCV infection, cirrhosis, TNM stage, tumor size, histology, number of tumors, vascular invasion, nor survival. Both beta-catenin and $\mathrm{p} 53$ expression showed no significant association with ARID1A. By immunohistochemistry of the whole sections, four (36\%) of 11 cases 